\title{
Study of a sample of faint Be stars in the exofield of CoRoT
}

\section{Spectroscopic characterization ${ }^{\star}$}

\author{
T. Semaan ${ }^{1}$, A. M. Hubert ${ }^{1}$, J. Zorec ${ }^{2}$, C. Martayan ${ }^{3,1}$, Y. Frémat ${ }^{4}$, J. Gutiérrez-Soto ${ }^{5,6,7}$, and J. Fabregat ${ }^{8}$ \\ 1 GEPI, Observatoire de Paris, CNRS UMR 8111, Université Paris Diderot, Place Jules Janssen, 92195 Meudon, France \\ 2 Université Pierre \& Marie Curie, CNRS UMR 7095, Institut d'Astrophysique de Paris, 98bis Bd. Arago, 75014 Paris, France \\ ${ }^{3}$ European Organization for Astronomical Research in the Southern Hemisphere, Alonso de Cordova 3107, Vitacura, \\ Santiago de Chile, Chile \\ 4 Royal Observatory of Belgium, 3 avenue Circulaire, 1180 Brussels, Belgium \\ 5 Instituto de Astrofisica de Andalucia (CSIC), Glorieta de la Astronomia s/n 18008, Granada, Spain \\ ${ }^{6}$ LESIA, Observatoire de Paris, CNRS UMR 8109, UPMC, Université Paris Diderot, 5 place Jules Janssen, 92195 Meudon, France \\ 7 Universidad Internacional Valenciana - VIU, Prolongación C/ José Pradas Gallen, s/n Edificio B 2 Piso, \\ 12006 Castellón de la Plana, Spain \\ 8 Observatorio Astronómico de la Universidad de Valencia, Calle Catedrático Agustín Escardino 7, 46980 Paterna, Valencia, Spain
}

Received 10 February 2012 / Accepted 2 November 2012

\begin{abstract}
Context. Be stars are probably the most rapid rotators among stars in the main sequence (MS) and, as such, are excellent candidates to study the incidence of the rotation on the characteristics of their non-radial pulsations, as well as on their internal structure. Pulsations are also thought to be possible mechanisms that help the mass ejection needed to build up the circumstellar disks of Be stars.

Aims. The purpose of this paper is to identify a number of faint Be stars observed with the CoRoT satellite and to determine their fundamental parameters, which will enable us to study their pulsation properties as a function of the location in the HR diagram and to search for correlations with the light outbursts, which are possibly produced by discrete mass ejections.

Methods. We identified those objects in the exofields of CoRoT presenting the Be phenomenon using $\mathrm{H} \alpha$ surveys, as well as automated methods based on pulsation properties that we finally confirmed with FLAMES/GIRAFFE and X-shooter spectroscopic observations at VLT/ESO, and with near-IR photometry. The spectra were 1) corrected for the veiling effect, 2) treated with the GIRFIT code to determine apparent fundamental parameters, and 3) corrected with the FASTROT code for effects induced by the rapid rotation.

Results. A list of $41 \mathrm{Be}$ star candidates were found from photometric and spectroscopic criteria. The spectral coverage useful for determining the fundamental parameters was obtained for only about half of them. We then spectroscopically identified 21 Be stars, two probable Be stars, and two B stars contaminated by the Sh 2-284 nebulosity. A short description of the spectral characteristics of each star is given. The fundamental parameters and, in particular, the rotation frequency $v_{\mathrm{r}}$ (cycles per day) were all corrected for rotational effects at rotation rates ranging from $\Omega / \Omega_{\mathrm{c}}=0.8$ to 1.0 . We have determined the positions of Be stars in the HR diagram and find two of them located beyond the MS phase.

Conclusions. The well-determined fundamental parameters presented in this paper for a statistically reliable sample of Be stars will enable us in forthcoming papers to compare the properties of stars as pulsators either according to their location in different regions of the HR diagram or with those predicted from theory.
\end{abstract}

Key words. stars: emission-line, Be - stars: early-type - stars: fundamental parameters - binaries: spectroscopic - stars: rotation stars: oscillations

\section{Introduction}

The CoRoT mission (COnvection, ROtation and planetary Transits, Baglin et al. 2006) has allowed observation and discovery of a large number of variable stars belonging to different classes. Among hot pulsating stars we focus on Be stars, which share the instability domain of classical B pulsating stars $(\beta$ Cephei and Slowly Pulsating B stars, hereafter SPB stars) in the HR diagram (Hertzsprung-Russell). Unlike these classical pulsators, Be stars are very rapid rotators and they are surrounded by an equatorially concentrated envelope or

\footnotetext{
* Appendices are only available in electronic form at http://www. aanda.org
}

decretion disk, possibly fed by mass-loaded winds produced by the interaction of continuous and variable stellar winds with discrete mass loss ejecta from the central star, as discussed in Moujtahid et al. (1999), Zorec et al. (2000), Hubert et al. (2000), and Meilland et al. (2006). However, the rotational angular velocity in Be stars does not reach the break-up velocity (Frémat et al. 2005). Nonradial pulsations (NRP) with transfer of angular momentum could be one of those efficient mechanisms that drive these stars at the limit of stability recurrently and produce mass loss events with associated light outbursts, such as those found by Hubert \& Floquet (1998), Mennickent et al. (2002), and Keller et al. (2002). Therefore, Be stars are good laboratories for studying, on one hand, the impact of rotation on pulsations, 
on the other, the role of pulsation in the recurrent mass ejection phenomena. A small sample $(<10)$ of bright Be stars has been observed in the seismology field of CoRoT showing multiple periods due to pulsation modes (Gutiérrez-Soto et al. 2011). Among these stars, HD 49330 displayed a light outburst. In this early B-type star, Huat et al. (2009) find that frequencies and variations in amplitude of pulsation modes change during all the different phases of the light outburst. To investigate the relation between rapid rotation, pulsations, and mass loss events in Be stars, accurate stellar fundamental parameters are required. This has been done for Be stars preselected as possible targets in the seismology field of CoRoT (Frémat et al. 2006).

To investigate the global properties of pulsation modes in Be stars with and without light outbursts, we need to consider a significant sample. To this end we turned towards exofields of CoRoT in which about 10000 faint stars $(11<V<17)$ were observed in each run. The CoRoT IR1 (Initial Run), LRC1, and LRA1 (Long Runs) exoplanet fields are areas defined on the sky where CoRoT has observed. The IR1 field is located towards the direction of the Monoceros constellation (hereafter called "field directed towards the galactic anticenter"), which contains stars within the coordinates $06^{\mathrm{h}} 43^{\mathrm{m}} 10^{\mathrm{s}}<\alpha<06^{\mathrm{h}} 50^{\mathrm{m}} 47^{\mathrm{s}}$ and $-03^{\circ} 20^{\prime} 00^{\prime \prime}<\delta<-00^{\circ} 25^{\prime} 00^{\prime \prime}$. In this field, 9879 stars were observed during 55 days from 6 February to 2 April 2007. The LRC1 field is located towards the Aquilae constellation (hereafter called "field directed towards the galactic center"), which contains stars within the coordinates $19^{\mathrm{h}} 22^{\mathrm{m}} 00^{\mathrm{s}}<\alpha<$ $19^{\mathrm{h}} 31^{\mathrm{m}} 30^{\mathrm{s}}$ and $-00^{\circ} 42^{\prime} 00^{\prime \prime}<\delta<+02^{\circ} 18^{\prime} 30^{\prime \prime}$. The LRC1 field corresponds to the first long run, where 11448 stars were observed during 153 days from the 16 May to 15 October 2007. The LRA1 field is located near the IR1 and the two fields have a common area part. The LRA1 contains stars within the coordinates $06^{\mathrm{h}} 40^{\mathrm{m}} 10^{\mathrm{s}}<\alpha<06^{\mathrm{h}} 46^{\mathrm{m}} 30^{\mathrm{s}}$ and $-01^{\circ} 37^{\prime} 00^{\prime \prime}<\delta<$ $+01^{\circ} 07^{\prime} 00^{\prime \prime}$ and 11448 stars were observed during 132 days from 23 October 2007 to 3 March 2008. Our knowledge of the spectral type and spectral properties of the faint stars already observed is most often fairly fragmentary in the range of magnitudes attainable in the CoRoT exofield.

The present study is dedicated to identifying Be stars in the first exofields of CoRoT (IR1, LRA1, LRC1) and to their spectroscopic characterization. We first present the setting up of our sample of faint Be stars (Sect. 2). We report in Sect. 3 the observation and the reduction of spectra obtained with the multifiber FLAMES/GIRAFFE spectrograph (Pasquini et al. 2002), as well as additional observations with the single-target X-shooter spectrograph (D'Odorico et al. 2006; Vernet et al. 2011) installed at the VLT-UT2 at ESO. In Sect. 4 we give spectral characteristics of the sample and in Sect. 5 we analyze the color-color diagram based on 2MASS data. Then in Sect. 6 we determine fundamental parameters taking fast rotation and veiling effects into account.

\section{The setting up of the faint Be-star sample}

The sample of our B emission-line stars was established thanks to three campaigns whose observations were carried out with different instruments and techniques before and after the launch of the CoRoT satellite. A photometric campaign in the Strömgren uvby system was conducted before the launch of the CoRoT satellite (Gutiérrez-Soto 2006). A first run was made in 2003 with the $1.5 \mathrm{~m}$ telescope of the Observatorio Astronómico Nacional (OAN) in Calar Alto, Almería, Spain. A sample of emission-line stars from the catalogs by Kohoutek \& Wehmeyer (1999, 2003, 2004) and Robertson \& Jordan (1989) was observed. The targets were selected on the base of spectral types determined considering their position in the reddening-free $\left[m_{1}\right]-\left[c_{1}\right]$ photometric diagram (Moon 1986).

A second campaign was carried out in 2005 with the $2.5 \mathrm{~m}$ Isaac Newton telescope at the Observatorio del Roque de los Muchachos (ORM), La Palma, Spain, equipped with the Wide Field Camera (Gutiérrez-Soto 2006). Observations were done through the Strömgren $u v b y$, the Sloan $r$, and an additional narrow filter centered on the $\mathrm{H} \alpha$ line. We used three nights in August and three in December to cover the LRA1 and LRC1 fields, respectively. Two exposure times were used for each filter and in both CoRoT fields, to ensure a more uniform $\mathrm{S} / \mathrm{N}$ for stars whose magnitudes range from 11 to $17 \mathrm{mag}$. Identification of Be stars was done in a two-stage process. In the first stage, the $(r-\mathrm{H} \alpha)-(b-y)$ photometric diagram was used to select emission line stars, which show up above a narrow sequence defining the locus of the absorption-line stars. In the second stage, the $\left[m_{1}\right]-\left[c_{1}\right]$ photometric diagram was used to determine the spectral type of the emission-line stars detected, as described above. We obtained spectral types from the Strömgren photometry as described above, which are given in Table 1 (Gutiérrez-Soto 2006). We also selected emission-line stars in the CoRoT exoplanetary fields from the IPHAS Catalogue of $\mathrm{H} \alpha$ Emission Line Sources in the Northern Galactic Plane (Witham et al. 2008).

In addition, low-resolution spectra were obtained in 2008 for several stars (see Table 1) at different observatories in Spain: the Observatorio de Sierra Nevada (OSN) with the Albireo spectrograph attached to the $1.5 \mathrm{~m}$ telescope, the Centro Astronómico Hispano Alemán in Calar Alto with the Cafos spectrograph attached to the $2.2 \mathrm{~m}$ telescope, and the Observatorio del Roque de los Muchachos with the Intermediate Dispersion Spectrograph (IDS) attached to the Isaac Newton telescope (INT). These spectra were obtained to identify new Be stars in the exoplanet fields and to provide a rough spectral classification for them.

A second study also took place before the launch of CoRoT and was performed in the framework of the guaranteed time attributed to the Observatoire de Paris, and carried out with the multiobject spectrograph VLT/FLAMES at ESO (PI, F. Hammer $)^{1}$. These observations, carried out during only two halfnights, were intended for spectral classification of stars covering the HR diagram and for identifying objects in exoplanet fields of CoRoT having specific characteristics, such as chemically peculiar HgMn objects and O/B/A emission line stars. A small fraction of stars could be observed in the LRA1 (Jan. 2005) and in the LRC1 fields (May 2005). Only two Be stars out of 200 stars were observed in the LRA1, and not a single one was detected in the LRC1 field.

A third and more exhaustive campaign (PI, C. Neiner) was undertaken in 2009 and 2010 with the VLT/FLAMES instrumentation $^{2}$ after the launch of the satellite. The goal of this observational program was multiple, although we have mostly focused on identifying Be stars and determining their fundamental parameters.

Thanks to photometric and spectroscopic studies, we constituted a sample of 41 emission-line stars that are listed in Table 1, but only 38 of them are Be stars as shown in Sects. 4 and 5 . We note that by using FLAMES/GIRAFFE and X-shooter spectra we also identified (or confirmed) 17 Be stars, two potentially Be star candidates, two B stars contaminated by nebulosity attributed to Sharpless 2-284, and one cool star, probably a T Tauri star as shown in Sects. 4 and 5. Moreover,

\footnotetext{
Program ID: 074.D-0193(A) and 075.D-0221(A).

Program ID: 082.D-0839(A) and 083.D-0479(A).
} 
T. Semaan et al.: Study of a sample of faint Be stars in the exofield of CoRoT. I.

Table 1. Be star candidates in the first exofields of CoRoT.

\begin{tabular}{|c|c|c|c|c|c|c|}
\hline $\begin{array}{l}\text { CoRoT ID } \\
-\end{array}$ & $\begin{array}{c}\text { Run } \\
-\end{array}$ & $\begin{array}{c}V \\
\text { mag }\end{array}$ & $\begin{array}{c}r \\
\mathrm{mag}\end{array}$ & $\begin{array}{c}\text { Spec. type } \\
-\end{array}$ & $\begin{array}{c}\text { Photometry } \\
-\end{array}$ & $\begin{array}{c}\text { Spectroscopy } \\
-\end{array}$ \\
\hline 101486436 & LRC1 & 12.333 & 12.237 & - & $\overline{-}$ & GIRAFFE \\
\hline 102595654 & LRA1 & 13.819 & 13.522 & - & - & GIRAFFE \\
\hline 102613778 & LRA1 & 15.409 & 15.096 & - & - & GIRAFFE \\
\hline 102617376 & LRA1 & 12.597 & 12.226 & - & T1.5-OAN & $\mathrm{R} \& \mathrm{~J}$ \\
\hline 102638133 & LRA1 & 14.852 & 14.462 & B3 & INT & - \\
\hline 102640224 & LRA1 & 15.324 & 15.125 & B9 & INT & - \\
\hline 102645018 & LRA1 & 13.214 & 12.718 & B0 & INT & $\mathrm{R} \& \mathrm{~J}$ \\
\hline 102649238 & LRA1 & 13.959 & 13.576 & A0 & INT & - \\
\hline 102656190 & LRA1 & 13.885 & 13.706 & B3 & INT & GIRAFFE \\
\hline 102667801 & LRA1 & 13.167 & 13.012 & B5 & INT & - \\
\hline 102672979 & LRA1 & 11.473 & 11.337 & - & - & GIRAFFE \\
\hline 102675453 & LRA1 & 14.887 & 14.727 & B3 & INT & - \\
\hline 102686433 & LRA1 & 13.475 & 13.253 & - & - & GIRAFFE \\
\hline 102690931 & LRA1 & 14.041 & 13.853 & B5 & INT & - \\
\hline 102695055 & LRA1 & 16.367 & 15.859 & - & - & GIRAFFE \\
\hline 102695698 & LRA1 & 13.914 & 13.758 & - & - & GIRAFFE \\
\hline 102696078 & LRA1 & 12.840 & 12.668 & - & - & $R \& J$ \\
\hline 102710394 & LRA1 & 14.459 & 14.128 & B8 & INT & - \\
\hline 102719279 & LRA $1 *$ & 14.074 & 13.809 & B9 & INT & CAFOS, GIRAFFE \\
\hline 102721109 & LRA1* & 15.505 & 15.035 & - & - & GIRAFFE \\
\hline 102725623 & LRA $1 *$ & 15.165 & 14.820 & B6 & INT & CAFOS, GIRAFFE \\
\hline 102728404 & LRA1 & 11.928 & 11.604 & - & - & R\&J, GIRAFFE \\
\hline 102736797 & LRA1 & 12.873 & 12.678 & B2 & INT & - \\
\hline 102737893 & LRA1 & 12.266 & 11.915 & - & - & $\mathrm{R} \& \mathrm{~J}$ \\
\hline 102749262 & LRA1 & 15.864 & 15.599 & - & - & GIRAFFE \\
\hline 102749390 & LRA1 & 14.120 & 13.906 & B3 & INT & - \\
\hline 102761769 & IR1 & 13.288 & 13.080 & - & - & IDS \\
\hline 102762536 & LRA1 & 13.902 & 13.513 & B0 & INT & - \\
\hline 102766835 & LRA1* & 13.197 & 12.817 & B2 & T1.5-OAN & R\&J, CAFOS, GIRAFFE \\
\hline 102777669 & LRA1 & 14.640 & 14.391 & - & - & GIRAFFE \\
\hline 102781703 & LRA1 & 12.663 & 12.314 & B3 & INT & - \\
\hline 102785204 & LRA1 & 12.058 & 11.756 & B0 & INT & R\&J, GIRAFFE, X-shooter \\
\hline 102785480 & LRA1 & 12.899 & 12.591 & B2 & INT & R\&J, GIRAFFE \\
\hline 102791482 & IR1 & 12.908 & 12.596 & - & - & CAFOS \\
\hline 102798228 & IR1 & 15.287 & 15.001 & - & - & ALBIREO \\
\hline 102825808 & IR1 & 14.424 & 14.165 & - & - & ALBIREO, GIRAFFE \\
\hline 102829773 & IR1 & 15.211 & 14.836 & - & - & $\mathrm{X}$-shooter \\
\hline 102847615 & IR1 & 13.873 & 13.572 & - & - & GIRAFFE \\
\hline 102875251 & IR1 & 14.799 & 14.536 & - & - & ALBIREO, GIRAFFE \\
\hline 102904910 & IR1 & 14.019 & 13.773 & - & - & CAFOS, X-shooter \\
\hline 102964342 & IR1 & 14.550 & 14.256 & - & - & CAFOS \\
\hline
\end{tabular}

Notes. (*) Observed by CoRoT in the LRA1 and IR1. Columns 3 \& 4: magnitudes from the Exo-Dat database (Deleuil et al. 2009). Column 5: spectral types determined from Strömgren photometry. Column 6: telescopes used to identify photometrically the stars with the Be phenomenon. Column 7: instruments that helped identify the Be stars spectroscopically. GIRAFFE stands for the multifiber spectrograph at ESO; X-shooter for the single target spectrograph at ESO; INT for the Isaac Newton Telescope in la Palma (Spain); T1.5-OAN for telescope of $1.5 \mathrm{~m}$ in Observatorio Astronomico Nacional (OAN) in Calar Alto, Almeria (Spain); ALBIREO for the low resolution spectrograph at Observatorio de Sierra Nevada (OSN), Granada (Spain); CAFOS for the low resolution spectrograph at the Centro Astronómico Hispano Alemán (CAHA) in Calar Alto, Almeria (Spain); IDS for the Intermediate Dispersion Spectrograph attached on the Isaac Newton telescope (INT) at the Observatorio del Roque de los Muchachos in la Palma (Spain); R\&J indicates stars in the emission-line catalog of Robertson \& Jordan (1989).

from low-resolution spectra obtained in Spain we identified four Be stars. These stars are CoRoT: 102761769, 102791482, 102798228 , and 102964342.

\section{Observation and reduction of spectra}

For some Be stars low-resolution spectra were obtained in Spanish observatories. However, most of the Be stars were observed at medium resolution with FLAMES/GIRAFFE multifiber spectrograph, but three of them with well-established Be characteristics were also recently monitored with X-shooter, a second-generation instrument at the ESO VLT. Since observations and the reduction of spectra have different characteristics in both instruments, we present them separately.

\subsection{Low-resolution spectra}

\subsubsection{ALBIREO spectra}

The spectra of the stars CoRoT 102798228, 102825808, and 102875251 were obtained with the ALBIREO spectrograph on 23 December 2008. The spectrograph was used in wideslit mode ("Rendija larga"), which allowed us to observe 
Table 2. Log of the observed stars with FLAMES/GIRAFFE and X-shooter.

\begin{tabular}{|c|c|c|c|c|c|c|c|c|c|c|c|}
\hline CoRoT ID & $\mathrm{Nb}$ & $\overline{\mathrm{RA}_{\mathrm{J} 2000}}$ & $\overline{\text { Dec }_{\mathrm{J} 2000}}$ & Run & Grat & $\overline{\mathrm{F}}$ & EXP & DATE-OBS & 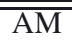 & $\overline{\mathrm{SSN}}$ & Moon \\
\hline \multirow{5}{*}{101486436} & \multirow{5}{*}{1} & \multirow{5}{*}{192903.13} & \multirow{5}{*}{ +003257.6 } & \multirow{5}{*}{ LRC1-1 } & LR2 & 1 & 2400 & 2009-06-09T04:15:45 & 1.47 & 116 & $94 \%$ \\
\hline & & & & & LR2 & 2 & 1179 & 2009-06-09Т04:57:21 & 1.29 & 115 & $94 \%$ \\
\hline & & & & & & 1 & 583 & $2009-05-26 \mathrm{~T} 09: 18: 36$ & 1.19 & 165 & $11 \%$ \\
\hline & & & & & LR6 & 2 & 583 & 2009-05-26T09:29:12 & 1.21 & 172 & $11 \%$ \\
\hline & & & & & & 3 & 133 & 2009-05-26T09:40:01 & 1.24 & 92 & $11 \%$ \\
\hline \multirow{3}{*}{102595654} & \multirow{3}{*}{2} & \multirow{3}{*}{064119.28} & \multirow{3}{*}{ +005659.2 } & \multirow{3}{*}{ LRA1-a } & LR2 & 1 & 2400 & 2009-02-10T02:45:22 & 1.13 & 82 & $96 \%$ \\
\hline & & & & & I R6 & 1 & 540 & 2009-02-10T03:36:51 & 1.21 & 67 & $96 \%$ \\
\hline & & & & & LKO & 2 & 540 & 2009-02-10T03:46:43 & 1.23 & 61 & $96 \%$ \\
\hline \multirow{3}{*}{102613778} & \multirow{3}{*}{3} & & & & & 1 & 540 & 2009-03-17T02:41:51 & 1.54 & 47 & $55 \%$ \\
\hline & & 064145.40 & +00 1436.1 & LRA1-d & LR6 & 2 & 800 & 2009-03-17T02:51:44 & 1.61 & 56 & $55 \%$ \\
\hline & & & & & & 3 & 418 & 2009-03-17T03:06:02 & 1.73 & 45 & $55 \%$ \\
\hline & & & & & IR? & 1 & 1800 & 2005-01-31T00:44:11 & 1.26 & 77 & $61 \%$ \\
\hline 102656190 & 4 & 064244.10 & -005102.1 & LRA1-X & LKL & 2 & 1800 & 2005-01-31T01:15:05 & 1.18 & 88 & $61 \%$ \\
\hline & & & & & LR6 & 1 & 2400 & 2005-01-31T01:56:37 & 1.12 & 122 & $61 \%$ \\
\hline & & & & & LR2 & 1 & 2400 & 2009-02-09T02:46:09 & 1.10 & 125 & $99 \%$ \\
\hline 102672979 & 5 & 064305.19 & -012304.0 & LRA1-q & IR6 & 1 & 540 & 2009-02-09T03:35:50 & 1.17 & 164 & $99 \%$ \\
\hline & & & & & LKO & 2 & 540 & 2009-02-09T03:45:43 & 1.19 & 158 & $99 \%$ \\
\hline & & & & & LR2 & 1 & 2400 & 2009-02-09T02:46:09 & 1.10 & 102 & $99 \%$ \\
\hline & & & & LRA1-q & I R6 & 1 & 540 & 2009-02-09T03:35:50 & 1.17 & 101 & $99 \%$ \\
\hline & & & & & LKO & 2 & 540 & 2009-02-09T03:45:43 & 1.19 & 96 & $99 \%$ \\
\hline 102686433 & 6 & 064321.97 & -012229.0 & & I P? & 1 & 1800 & $2005-01-31 \mathrm{~T} 02: 46: 53$ & 1.09 & 121 & $61 \%$ \\
\hline & & & & IRA & LK 2 & 2 & 1800 & 2005-01-31T03:17:47 & 1.10 & 116 & $61 \%$ \\
\hline & & & & & I R6 & 1 & 2400 & $2005-01-31 \mathrm{~T} 03: 57: 48$ & 1.15 & 167 & $61 \%$ \\
\hline & & & & & LKO & 2 & 600 & 2005-01-31T04:38:44 & 1.24 & 91 & $61 \%$ \\
\hline 102605055 & 7 & $06-132364$ & 0103207 & J P 1 n & I P6 & 1 & 540 & $2009-03-22 \mathrm{~T} 00: 54: 20$ & 1.18 & 37 & $12 \%$ \\
\hline 102095055 & 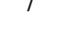 & 32.04 & 10320.1 & LKAI-n & LK6 & 2 & 540 & 2009-03-22T01:04:13 & 1.20 & 35 & $12 \%$ \\
\hline 102605608 & 8 & $06=12=2346$ & 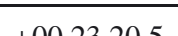 & I D 11 & $\mathrm{D} 6$ & 1 & 540 & 2009-02-09T04:10:28 & 1.28 & 93 & $99 \%$ \\
\hline 102095090 & & 00455.40 & $00<5<0.3$ & LRA1-e & LKO & 2 & 540 & 2009-02-09T04:20:21 & 1.32 & 95 & $99 \%$ \\
\hline 10771070 & 0 & $06=140424$ & 0041156 & $I D$ & LR2 & 1 & 2400 & 2009-01-13T02:46:21 & 1.14 & 125 & $87 \%$ \\
\hline 102719219 & 9 & 004404.24 & $0041 \quad 15.0$ & LKAI-K & LR6 & 1 & 540 & 2009-01-15T03:00:09 & 1.12 & 81 & $69 \%$ \\
\hline 102721109 & 10 & 064406.92 & -0101386 & $\mathrm{LPA} 1 \mathrm{n}$ & IR6 & 1 & 540 & $2009-03-22 \mathrm{~T} 00: 54: 20$ & 1.18 & 54 & $12 \%$ \\
\hline & & & & LNA1-11 & LKO & 2 & 540 & 2009-03-22T01:04:13 & 1.20 & 50 & $12 \%$ \\
\hline & & & & & LR2 & 1 & 2400 & 2009-02-09T02:46:09 & 1.10 & 65 & $99 \%$ \\
\hline 102725623 & 11 & 064413.41 & -012524.6 & LRA1-q & I R6 & 1 & 540 & 2009-02-09T03:35:50 & 1.17 & 61 & $99 \%$ \\
\hline & & & & & LKO & 2 & 540 & 2009-02-09T03:45:43 & 1.19 & 64 & $99 \%$ \\
\hline 102728404 & 12 & 06411748 & 7 & $\mathrm{~J} A \mathrm{~A} \boldsymbol{f}$ & J P? & 1 & 2400 & 2009-03-05Т02:29:21 & 1.26 & 171 & $72 \%$ \\
\hline 102128404 & 12 & 004417.48 & 31.1 & LKAI-I & LKL 2 & 2 & 2400 & 2009-03-05T02:29:21 & 1.26 & 101 & $72 \%$ \\
\hline 102749262 & 13 & 064445.49 & +001902.8 & LRA1-f & LR2 & 1 & 2400 & 2009-03-05Т02:29:21 & 1.26 & 30 & $72 \%$ \\
\hline 102766835 & 14 & 06450927 & -015206 & $\mathrm{JPA} 1-\mathrm{r}$ & LR2 & 1 & 2400 & 2009-01-08Т04:55:29 & 1.11 & 128 & $95 \%$ \\
\hline $102 / 00035$ & 14 & 004509.21 & $011 \quad 15<0.0$ & LNAI-1 & LR6 & 1 & 546 & 2009-01-08T05:44:15 & 1.17 & 149 & $95 \%$ \\
\hline 102777669 & 15 & 064523.88 & +002204.1 & LRA1-f & LR2 & 1 & 2400 & 2009-03-05T02:29:21 & 1.26 & 63 & $72 \%$ \\
\hline & & & & LRA1-f & LR2 & 1 & 2400 & 2009-03-05T02:29:21 & 1.26 & 118 & $72 \%$ \\
\hline 102785204 & 16 & 06453376 & 1 & & UVB & 1 & 120 & $2010-09-12 \mathrm{~T} 09: 15: 39$ & 1.48 & 90 & $29 \%$ \\
\hline $102 / 85204$ & 16 & 064533.16 & +002010.1 & LRA1-y & VIS & 1 & 140 & 2010-09-12T09:15:44 & 1.48 & 72 & $29 \%$ \\
\hline & & & & & NIR & 1 & 120 & 2010-09-12T09:15:49 & 1.48 & 14 & $29 \%$ \\
\hline 102785480 & 17 & 064534.14 & +003117.1 & LRA1-f & LR2 & 1 & 2400 & 2009-03-05T02:29:21 & 1.26 & 123 & $72 \%$ \\
\hline & & & & & I R? & 1 & 900 & $2010-01-26 \mathrm{~T} 00: 45: 30$ & 1.34 & 60 & $87 \%$ \\
\hline 102825808 & 18 & 06464370 & 0104284 & IP1 & LK2 & 2 & 900 & 2010-01-26T01:15:02 & 1.24 & 75 & $87 \%$ \\
\hline 102025000 & 10 & $00+045.10$ & 010420.4 & $1 K 1-e^{2}+2 y$ & I P & 1 & 558 & 2009-03-04T01:40:51 & 1.13 & 48 & $61 \%$ \\
\hline & & & & & LKO & 2 & 558 & 2009-03-04T01:51:02 & 1.14 & 45 & $61 \%$ \\
\hline & & & & & $\overline{\mathrm{UVB}}$ & 1 & 480 & 2010-09-16T09:03:42 & 1.41 & 33 & $70 \%$ \\
\hline 102829773 & 19 & 064650.49 & -030758.5 & IR1-y & VIS & 1 & 500 & 2010-09-16T09:03:47 & 1.41 & 52 & $70 \%$ \\
\hline & & & & & NIR & 1 & 480 & 2010-09-16T09:03:53 & 1.41 & 16 & $70 \%$ \\
\hline & & & & & $\mathrm{J}$ & 1 & 900 & $2010-01-26 \mathrm{~T} 00: 45: 30$ & 1.34 & 62 & $87 \%$ \\
\hline 102847615 & 20 & 06471885 & -011029 & IR1_- & $\mathrm{L}$ & 2 & 900 & 2010-01-26T01:15:02 & 1.24 & 77 & $87 \%$ \\
\hline & 20 & & 011029.9 & $1 K 1-e^{-2}+2$ & I & 1 & 558 & 2009-03-04T01:40:51 & 1.13 & 55 & $61 \%$ \\
\hline & & & & & LKO & 2 & 558 & 2009-03-04T01:51:02 & 1.14 & 54 & $61 \%$ \\
\hline 102875251 & 21 & 5818 & -0033 & IR & I R6 & 1 & 558 & 2009-03-04T02:58:08 & 1.32 & 58 & $61 \%$ \\
\hline 1020/J2J1 & 21 & 58.18 & & & LR6 & 2 & 558 & 2009-03-04T03:08:19 & 1.36 & 41 & $61 \%$ \\
\hline & & & & & UVB & 1 & 300 & 2010-09-14T09:39:07 & 1.32 & 56 & $50 \%$ \\
\hline 102904910 & 22 & 064837.47 & -005620.4 & IR1-y & VIS & 1 & 360 & 2010-09-14T09:39:12 & 1.32 & 43 & $50 \%$ \\
\hline & & & & & NIR & 1 & 300 & 2010-09-14T09:39:17 & 1.32 & 14 & $50 \%$ \\
\hline
\end{tabular}

Notes. Column 5 "Run" CoRoT run in upper case and GIRAFFE field in lower case. Column 6: "Grat." setup used with GIRAFFE and the arm used with X-shooter. Column 7: "F" number of the frame. Column 8: "EXP" exposure-time in seconds. Column 10: "AM" air mass. Column 11: "SN" signal to noise of spectra. Column 12: "Moon" percent of illumination due to the moon during the night. 
a wavelength domain between $3263 \AA$ and $7337 \AA$. The resolution is $R=1100$.

\subsubsection{IDS spectra}

CoRoT 102761769 is the only star that was observed with IDS (Intermediate Dispersion Spectrograph) on 30 March 2008. The R300V grating $(300 \mathrm{l} / \mathrm{mm})$ was used with a dispersion of $1.87 \mathrm{~A} / \mathrm{pix}$, and the spectral wavelength coverage is $\lambda \lambda$ 3337-8366 $\AA$. The spectra of the star 102761769 have already been analyzed by Emilio et al. (2010).

\subsubsection{CAFOS spectra}

CoRoT 102725623, 102791482, and 102964342 were observed with the CAFOS spectrograph on 11 March 2008. We used the B-200 grism with a dispersion of $4.7 \AA$ /pix and a spectral wavelength coverage of 3200-7000 A.

\subsubsection{Reduction}

All the low-resolution spectra from ALBIREO, CAFOS, and IDS spectrographs were extracted following standard reduction steps with the IRAF software, including bias and flat corrections, 1-D aperture extraction, wavelength calibration, and normalization of the continuum.

\subsection{FLAMES/GIRAFFE spectra}

We obtained spectra with the multifiber FLAMES/GIRAFFE spectrograph in the MEDUSA mode at medium resolution to identify the Be stars present in the exofields of CoRoT and to determine their fundamental parameters. The MEDUSA mode enabled us to locate 132 fibers to observe stars simultaneously in a 26'-wide sky field. Most fibers were attributed to CoRoT targets, but a small number, from four to eight fibers, were used to observe the sky to correct the stellar spectra from possible contaminations. Thanks to the rather large number of stars observed simultaneously, we could cover a significant part of each CoRoT exofield and characterize most of them. We observed 34 GIRAFFE fields located in the first exofields of CoRoT, of which IR1 and LRA1 are in the anticenter direction of the Galaxy, while LRC1 is towards the galactic center. The coordinates of each star and the dates of the observation are given in Table 2. The GIRAFFE fields are indicated by lower case letters in Col. 5 of this table.

The primary observational strategy was to get three spectra for each program star. One of them was obtained in the LR2 setup (396.4-456.7 $\mathrm{nm}$, hereafter blue spectrum) and the other two successive spectra in the LR6 setup (643.8-718.4 nm, hereafter red spectra). However, it was not possible to attain the primary goal intended for each GIRAFFE field, mainly for stars in the IR1 field of CoRoT, since we could obtain spectra only in the red domain for most of them. Details concerning the grating setups and frames for each GIRAFFE field are given in Table 2. The resolutions of our spectra are $R=6400$ in the blue domain and $R=8600$ in the red one.

The reduction of spectra was made with the ESO pipeline. We used successively different recipes: GIMASTERBIAS to remove the bias, GIMASTERFLAT to locate the fibers on the detectors and compute the normalized flat-field, GIWAVECALIBRATION to calculate the dispersion solution, and then GISCIENCE, which provides the spectra reduced for all GIRAFFE fields corrected from the bias and duly wavelength-calibrated. Thanks to implementing modern CCD detectors in the FLAMES/GIRAFFE instrumentation, corrections for "dark" signals were not needed. Finally, we used the IRAF $^{3}$ package to separate all the spectra contained in each GIRAFFE exposure and have corrected them for the heliocentric velocity. The $\mathrm{S} / \mathrm{N}$ of our spectra range from 30 to 172 ; see the details in Table 2 .

In the GIRAFFE fields, an additional spectrum can be imprinted on one of the stellar targets during exposures of long enough integration time. This spectrum is a combination of telluric absorption lines and the solar spectrum due to the scattered moonlight. Most of our program stars were observed at nearly full Moon. The fraction of scattered moonlight that contaminates our spectra is given in Table 2. Only the long-exposure spectra of the fainter stars needed to be corrected for the sky emission spectrum.

\subsection{X-shooter spectra}

In September 2010, the Be stars CoRoT 102785204, 102829773, and 102904910 were observed (PI, C. Martayan) with the $\mathrm{X}$-shooter spectrograph ${ }^{4}$, in three spectral domains:

- the UV-blue domain $\lambda \lambda$ 3000-5500 $\AA$ (UVB);

- the visual-red domain $\lambda \lambda$ 5500-10000 $\AA$ (VIS);

- the near-IR domain $\lambda \lambda$ 10000-25000 $\AA$ (NIR).

The spectral resolutions and characteristics are $R=9800$ (slit $=0.5^{\prime \prime}$, readout $100 \mathrm{KHz}$, bining $1 \times 1$ ) for the UVB, 18000 (slit $=0.4^{\prime \prime}$, readout $100 \mathrm{KHz}$, bining $1 \times 1$ ) for the VIS, and 10500 for the NIR (slit $=0.4^{\prime \prime}$, readout: nondestructive mode). The stars observed with X-shooter and their primary characteristics are given in Table 2.

To reduce the X-Shooter data, we used the X-shooter ESO pipeline (Modigliani et al. 2010) with esorex recipes. For each recipe, the parameters were adjusted to optimize the quality of the data reduction, and full details will be provided in Martayan et al. (in prep.). However, the general steps were as follows by using daytime calibrations.

- The bias level was corrected for the UVB and VIS arms with corresponding bias frames of the same readout modes as science data.

- The dark for the NIR arm was corrected with NIR dark frames with the same DIT $\times$ NDIT as science frames. In addition, the bad pixel map was created and those pixels were taken into account in the next steps.

- A first guess of the wavelength and spectrum/orders localization was obtained with the format-check and order-definition frames for each arm.

- The flat field correction was performed for each arm, and this step allows the limits of each order to be properly defined.

- A wavelength 2D map calibration for different positions in each order (to take the curvature of the orders into account) was obtained with $\mathrm{Th} / \mathrm{Ar}$ or $\mathrm{Xe} / \mathrm{Ar} / \mathrm{Ne} / \mathrm{Hg}$ frames obtained with a set of multipinholes.

- The shift in the spectral format between the daytime calibrations and the science observation was determined and corrected via the use of a set of attached flexure compensation AFC frames taken at the acquisition step of the science data.

3 IRAF is distributed by the National Optical Astronomy Observatories, which is operated by the Association of Universities for Research in Astronomy (AURA), Inc., under cooperative agreement with the National Science Foundation.

4 Program ID: 084.D-0142(A). 
- The tilt of the lines and the resolving power were determined with slit ARC frames.

- Finally, the science spectra were extracted from the combination of two individual exposures obtained in the nodding mode. The curvature of the orders was corrected with the information obtained in the previous steps, and the orders were merged using the error spectra. The extracted science spectrum was calibrated in wavelength as well. There is also a possibility of flux-calibrating the spectrum, but this approach was not used in this study and is therefore not described here.

\section{Frequency of Be stars and characteristics of the observed ones}

In this section we briefly present the results from the GIRAFFE fields, those regarding the IR1 and the LRA1 CoRoT exofields together, on the one hand, and on the other, the LRC1 CoRoT exofield. The separation is done simply because of the galactic center and anticenter orientation of the CoRoT fields.

\subsection{Anticenter direction: LRA1 and IR1 exofields}

The LRA1 and IR1 fields pointed towards the anticenter of the Galaxy across the Orion spur, the Perseus arm, and the outer Norma-Cygnus arm (Russeil 2003). In the LRA1 field we can find several open stellar clusters: Dolidze 25 is a young cluster $(\log t(\mathrm{yr})=6.75$, Puga et al. 2009); Dolidze 23 is a poorly studied cluster; NGC 2286 is an old cluster $(\log t(\mathrm{yr})=8.88$, WEBDA database ${ }^{5}$ ). In addition, there are several $\mathrm{H}$ II ionized regions in this field, the largest being Sh 2-284 (Puga et al. 2009). In the IR1 and LRA1 CoRoT runs we observed 16 GIRAFFE fields in 2009 and 2010, which represent 2070 stars observed spectroscopically over 19878 objects observed photometrically by CoRoT. We thus have spectra for about $10 \%$ of CoRoT targets, but most of them are in the red wavelength range, specifically, 1820 red spectra and 1132 blue spectra. We could then study the $\mathrm{H} \alpha$ line for most stars, and also the $\mathrm{H} \gamma$ and $\mathrm{H} \delta$ Balmer lines for half of them. It was then possible to identify $94 \mathrm{~B}$ stars, of which 16 objects are Be stars and two that are good Be candidates. The percentage of Be stars of $12<V<16.5$ mag in the LRA1 and IR1 exofields is $N(\mathrm{Be}) /[N(\mathrm{Be})+N(\mathrm{~B})] \simeq 20 \%$, which is close to the average value of $17 \%$ found by Zorec \& Briot (1997) for the average Galactic field stellar population brighter than $V=7.10 \mathrm{mag}$. However, in the Galaxy this percentage can change according to the local physical conditions, mainly chemical composition (Maeder et al. 1999), and/or properties of the environment, in particular clusters, streams, and associations, where the percentage of Be stars depends on cluster age (Fabregat \& Torrejón 2000). Surveys by McSwain \& Gies (2005), Wisniewski \& Bjorkman (2006), and Mathew et al. (2008) show lower percentages $(<10 \%)$ in most of young Galactic open clusters. However, some of these have a higher Be star content that is about $18 \%$ in Ber 87, Col 96, and NGC 1220, going up to 26\% in NGC 2345 (Mathew et al. 2008) or approaching 30\% in NGC 663 and NGC 7419 (Pigulski \& Kopacki 2000; Pigulski et al. 2001). Nevertheless, the percentages of Be stars are underestimated because the Be phases are not permanent and detection of the Be phenomenon with slitless spectroscopy or photometry is not complete, since stars with very faint emission characteristics cannot be identified.

The stars observed by CoRoT in the IR1 and LRA1 fields are shown in the magnitude-color diagram $(V, B-V)$ in Fig. 1.

\footnotetext{
5 http://www. univie.ac.at/webda/
}

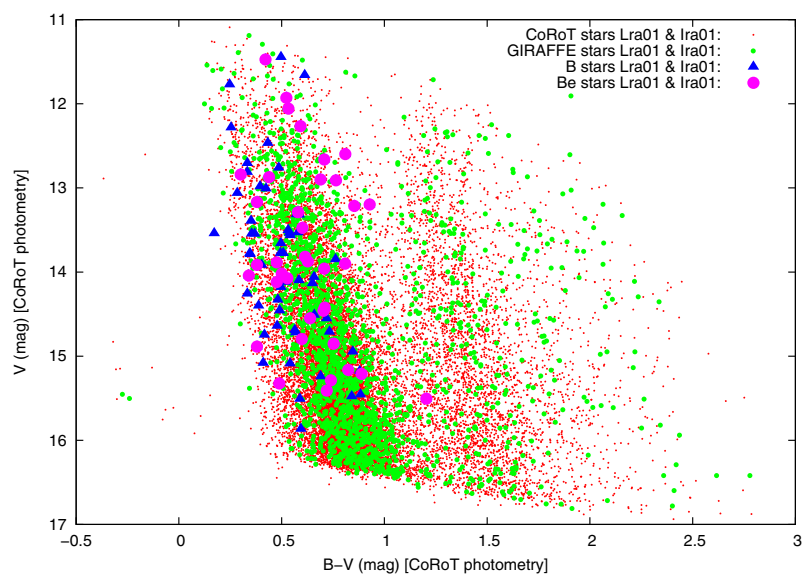

Fig. 1. Apparent magnitude-color diagram of the stars observed by CoRoT in the IR1-LRA1 runs and those for which we have obtained spectra with GIRAFFE. B and Be stars detected by spectroscopy are indicated.

The small red points are the targets observed with the satellite (19878 objects), which clearly depict a magnitude limited sample $\left(11 \lesssim V_{\text {obs }} \lesssim 17 \mathrm{mag}\right)$. The small green full circles represent the stars for which we have obtained spectra with the GIRAFFE spectrograph (1898 stars). Blue triangles are B-type stars, while the purple big full circles are Be stars.

\subsection{Center direction: LRC1 exofield}

The LRC1 field pointed towards the galactic center and contains no listed clusters of stars. In the LRC1 CoRoT run we observed 18 GIRAFFE fields, which encompass 2329 observed stars over 11408 objects observed photometrically by CoRoT. For $20 \%$ of our CoRoT targets we have both a blue and a red spectrum. From the analysis of red spectra, we find and/or confirm about ten stars as B-type stars, but from the study of Balmer lines we could find only one object that is a Be star. We readily notice that the number of B stars in the LRC1 exofield is very low compared to the number in the anticenter direction, contrary to what was expected from the preselection of B/Be-type stars made according to the adopted pulsation criteria. The fraction of Be stars amounts to about $10 \%$, but this percentage cannot be considered reliable because of the generally low number of B-type stars detected. The observed stars by CoRoT in the LRC1 field are shown in Fig. 2. In this figure the symbols and color codes are the same as for Fig. 1.

\subsection{Comments on the LRA1, IR1, and LRC1 exofields}

The low number of $\mathrm{B}$ and Be stars found in the LRC1 compared to the one in LRA1 and IR1 can be explained in terms of differences in the amount of the interstellar medium (hereafter, ISM) extinction in the two directions. The extinction is higher towards the galactic center (Drimmel et al. 2003; Sale et al. 2009), which leads to seeing so few B and Be stars. Towards the galactic center, we see a volume-limited sample of stars, as we cannot reach beyond $3 \mathrm{kpc}$ because of the high ISM extinction. However, it is important to keep in mind that the exoplanet star candidates selected for CoRoT are in the majority cool stars (spectral types later than A). Therefore only a small part of the stars selected for CoRoT in the IR1, LRA1 and LRC1 fields are $\mathrm{B} / \mathrm{Be}$ star candidates according to photometric measurements. 
T. Semaan et al.: Study of a sample of faint Be stars in the exofield of CoRoT. I.

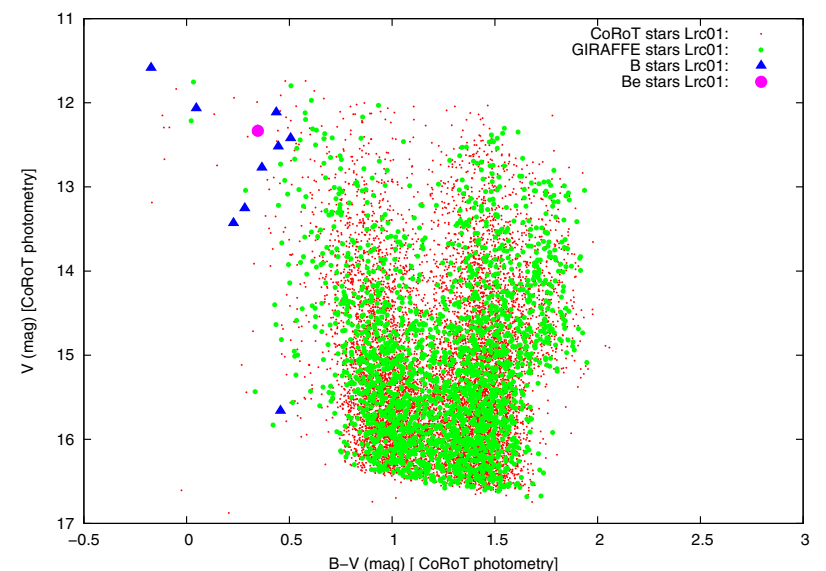

Fig. 2. Apparent magnitude-color diagram of the stars observed by CoRoT in the LRC1 run and those for which we have obtained spectra with GIRAFFE. B and Be stars detected by spectroscopy are indicated.

As a consequence we might not draw hasty conclusions on the proportions of B stars in these fields. We note that, if the CoRoT sample was not spectral type-biased, we could have had better chances to observe a significant larger number of B and Be-type stars.

\subsection{Spectral characteristics of the Be stars observed with VLT/UT2}

Spectra obtained at VLT/ESO enabled us to identify and/or confirm the $\mathrm{Be}$ character of stars located in the first exoplanetary fields observed by CoRoT. Moreover, they indicated the presence of lines formed in the circumstellar environments that sometimes make it quite difficult to determine the stellar fundamental parameters. For Be stars monitored with FLAMES/GIRAFFE, blue and red spectra are not always available for each target. Thus, for several stars, we only obtained spectra in the LR6 setup (wavelength range containing the $\mathrm{H} \alpha$ line). Depending on the photospheric lines that we could observe in this domain, we have not always been able to conclude whether the star is a Be star or not. At that point, the star is considered as a Be candidate.

We present a short description of the spectral characteristics of each Be star and three other ex-Be-stars candidates that are not confirmed by our analyses. Several quantities related to the circumstellar contribution in the first terms of the Balmer series, when available, such as the intensity and the radial velocity of the single-peaked or double-peaked (red and blue peaks, hereafter $R$ and $V$ ) emission and of a shell component (S), are given in Table 3. The $\mathrm{H} \alpha$ line profiles of Be stars are shown in Fig. 3. Narrow and broad absorption lines due to diffuse interstellar bands (DIBs) are seen in spectra. Among those we could identify in the visible and near-IR wavelength ranges are $\lambda \lambda 4232$, 4430, 4501, 6614, 6660, 6672, 6699, 6702, 6843, 6852, 6993, and 7069 (Galazutdinov et al. 2000).

CoRoT Id 101486436: a faint, double-peaked emission is centrally superposed on the photospheric $\mathrm{H} \alpha$ line profile. No emission is detected in blue lines.

CoRoT Id 102595654: for this fainter star, the spectrum was corrected for solar lines due to the full Moon in the sky at the epoch of the observation. $\mathrm{H} \alpha$ shows an asymmetrical double-peaked emission $(V<R)$ centrally superposed on the photospheric line profile. No emission was detected in the blue spectrum.

CoRoT Id 102613778: H $\alpha$ shows a weak, double-peaked emission, with a centrally deep and sharp absorption (shell feature), superposed on the core of the photospheric line profile. Red He I lines $\lambda \lambda 6678$ and 7065 are conspicuous, which indicates an effective temperature $T_{\text {eff }}>15000 \mathrm{~K}$. No blue spectrum is available.

CoRoT Id 102656190: $\mathrm{H} \alpha$ shows a strong double-peaked emission. The photospheric $\mathrm{H} \gamma$ line is disturbed by a weak doublepeaked emission.

CoRoT Id 102672979: H $\alpha$ shows a strong, single-peaked emission line. The photospheric $\mathrm{H} \gamma$ line is disturbed by a weak emission in its core. The same is observed for $\mathrm{H} \delta$ to a lesser degree.

CoRoT Id 102686433: $\mathrm{H} \alpha$ shows a double-peaked emission with a central reversal. A very weak, symmetrical emission is present on each side of the core of the photospheric $\mathrm{H} \gamma$ line profile. We note that emission is stronger in 2009 than in 2005.

CoRoT Id 102695055: H $\alpha$ shows a nearly symmetrical $(V \gtrsim R)$, double-peaked emission. The presence of the He I line is uncertain because the red spectrum is very noisy and no blue spectrum is available. According to 2MASS data analysis (see Sect. 4) this star is not a Be star.

CoRoT Id 102695698: $\mathrm{H} \alpha$ displays a symmetrical, doublepeaked emission in the core of the photospheric line profile. This star is probably a late Be star.

CoRoT Id 102719279: in the red domain, $\mathrm{H} \alpha$ displays a doublepeaked emission, which is highly asymmetrical $(V \ll R)$ and disturbed by a deep and sharp shell feature $\left(\mathrm{RV}=+70 \mathrm{~km} \mathrm{~s}^{-1}\right)$. The He I lines $\lambda \lambda 6678$ and 7065 are in absorption and very conspicuous. In the blue domain, only the red emission component $\left(\mathrm{RV}=+170 \mathrm{~km} \mathrm{~s}^{-1}\right.$ ) is seen in $\mathrm{H} \gamma$ and $\mathrm{H} \delta$; a strong $\mathrm{H}$ shell is also observed with $\mathrm{RV}=+30 \mathrm{~km} \mathrm{~s}^{-1}$. We note the presence of a metallic shell, mainly Fe II and Si II lines, with $\mathrm{RV}=+17 \mathrm{~km} \mathrm{~s}^{-1}$.

CoRoT Id 102721109: the star was only observed in the red domain. H $\alpha$ depicts a double-peaked emission, which is nearly symmetrical $(V \geq R)$, superimposed on a broad absorption. The spectrum is very noisy and many DIBS are present, however the He I lines are present. This object is considered to be a Be star with an effective temperature $T_{\text {eff }}>15000 \mathrm{~K}$.

CoRoT Id 102725623: for this other fainter star, the spectrum was corrected from solar lines due to the full Moon in the sky at the epoch of the observation. A shell is present on Balmer lines. $\mathrm{H} \alpha$ is a symmetrical double-peaked emission.

CoRoT Id 102728404: among Be stars of the sample, this one displays the stronger circumstellar contamination in its blue spectrum (no red spectra available). Strong and narrow emission 
substantially affects the core of the $\mathrm{H} \gamma$ and $\mathrm{H} \delta$ photospheric lines; moreover, the stronger Fe II lines of the multiplets 27, 28, 37,38 are present in emission that highly pollutes the He I and $\mathrm{Mg}$ II lines commonly used for determining stellar fundamental parameters.

CoRoT Id 102749262: this fainter star is located in the LRA1f field affected by Sh 2-284 ambient nebulosity. The very weak emission observed in the core of blue $\mathrm{H}$ photospheric lines is attributed to this nebulosity. The star is not considered a Be star.

CoRoT Id 102766835: the star is a late Oe star. The photospheric line He II $4200 \AA$ is very conspicuous. $\mathrm{H} \alpha$ is in emission; it shows a complex, asymmetrical (blue-winged) line profile with two peaks having similar intensity, separated by sharp absorption (shell) with $\mathrm{RV}=+118 \mathrm{~km} \mathrm{~s}^{-1}$. A secondary and very weak shell component seems to affect the blue wing of emission with $\mathrm{RV}=0 \mathrm{~km} \mathrm{~s}^{-1}$. Blue spectra also display strong circumstellar contamination. Emission affects the red wing of photospheric Balmer lines, and a shell component $\left(\mathrm{RV}=+7 \mathrm{~km} \mathrm{~s}^{-1}\right)$ is also present on those lines. Shell metallic lines (Fe II, Si II, Ni II) are observed with $\mathrm{RV}=+2 \mathrm{~km} \mathrm{~s}^{-1}$, the same value as the one of the secondary shell component in $\mathrm{H} \alpha$. The star could be a multiple system with a circumbinary envelope.

CoRoT Id 102777669: this star is another fainter object located in the LRA1f field. The asymmetry depicted in the core of Balmer lines is due to a very weak emission, which we attribute to the Sh 2-284 ambient nebulosity. The star is not considered to be a Be star.

CoRoT Id 102785204: only observed in the blue domain in 2009 with FLAMES/GIRAFFE, this early-B type star displays a very complex spectrum. Balmer and He I absorption lines are flanked by a narrow and conspicuous emission $\left(\mathrm{RV}=-40 \mathrm{~km} \mathrm{~s}^{-1}\right)$ in their blue wing. The RV of He I absorption lines is $+170 \mathrm{~km} \mathrm{~s}^{-1}$, while the one of metallic shell lines is $+220 \mathrm{~km} \mathrm{~s}^{-1}$. He II lines are also present, but their RV is uncertain. Higher dispersion is needed to explore the complex spectrum of this star. Fortunately, the star could be observed again in 2010 with X-shooter, which allows us to obtain a large wavelength coverage in the visual and near-IR ranges. We then note the presence of the photospheric lines (He II, C III etc.) typical of a very early $\mathrm{Be}$ star. $\mathrm{H} \alpha$ shows a double-peaked emission, which is asymmetrical $(R \gg V)$ with a shell at $\mathrm{RV}=+33 \mathrm{~km} \mathrm{~s}^{-1}$. Other Balmer lines present the same structure as in $\mathrm{H} \alpha$ with an emission contribution decreasing in intensity across the series. We note a change in asymmetry in H I circumstellar lines and in He I lines as compared to FLAMES/GIRAFFE observations in 2009, as well as a decrease in the emission strength. Stronger He I lines are disturbed by emission $\left(\mathrm{RV}=+155 \mathrm{~km} \mathrm{~s}^{-1}\right)$, and they also show a shell component $\left(\mathrm{RV}=+30 \mathrm{~km} \mathrm{~s}^{-1}\right)$ close to the one measured in Balmer lines. He I 10830 is a strong asymmetrical emission line ( $\operatorname{Imax}=2.8 ; R \gg V$ ) also disturbed by a deep and narrow shell component. Numerous Fe II emission lines with the same asymmetrical structure as the ones in $\mathrm{H}$ and $\mathrm{He} \mathrm{I}$ lines are present. Emission is seen up to H12 in the Balmer series, P25 in the Paschen series, and B24 in the Brackett series. Near-IR O I lines are in emission. The change in asymmetry in circumstellar profiles observed in 2010 as compared to FLAMES/GIRAFFE observations in 2009 could be due to a density wave propagating in the envelope/disk as often reported in classical Be stars (Okazaki 1991, 1997). Moreover, this star is probably a member $(p=65 \%)$ of the cluster Dolidze 25 according to Dias et al. (2006).

CoRoT Id 102785480: this star was previously checked as a Be star from photometry. Only a blue spectrum is available and we do not detect any emission in this spectral domain.

CoRoT Id 102825808: photospheric Balmer lines show a complex structure in their core, which could be explained by a weak emission, pulsations, or a binary. Moreover, this peculiar star has a high $v \sin i$.

CoRoT Id 102829773: the star was observed with X-shooter in 2010. $\mathrm{H} \alpha$ shows an emission line with a complex (maybe triple) structure. $\mathrm{H} \beta$ presents a double-peaked $(V \gg R)$ emission. For higher Balmer lines, only the $\mathrm{V}$ emission component is detected (up to H8). Higher terms of the Paschen and Brackett series (up to $\mathrm{P} 24$ and B22 respectively) are in double emission with $V \sim R$. Strong red He I lines are affected by emission; while He I 10830 is a double-peaked emission with $V>R$, only the $V$ emission component is seen in other red lines. We note the presence of Fe II emission lines with a double structure. O I 8446 is also in emission.

CoRoT Id 102847615: $\mathrm{H} \alpha$ is a very strong emission line. The photospheric $\mathrm{H} \gamma$ line shows a double-peaked emission in its core.

CoRoT Id 102875251: H $\alpha$ shows a symmetrical double-peaked emission line superposed on the photospheric line. He I 6678 is present, suggesting an effective temperature $T_{\text {eff }}>15000 \mathrm{~K}$.

CoRoT Id 102904910: the star was observed with X-shooter. $\mathrm{H} \alpha$ shows a symmetrical double-peaked emission with a central shell. Blue Balmer lines up to $\mathrm{H} \epsilon$ show the same emission structure in their photospheric component. Emission is also present in the Paschen and Brackett series. O I 8446 is in emission.

\section{IR color diagram of the studied Be stars}

In Be stars there is an IR flux excess produced by electron scattering, free-free, and free-bound radiation produced in their circumstellar disk. We studied this flux excess only in those Be stars for which we had observed spectra at intermediate and high resolutions. In these spectra we measured the equivalent width of two diffuse interstellar bands at $\lambda 4430 \AA$ and $\lambda 6613 \AA$ A. Using the calibrations of these bands into the color excess $E(B-V)$ (Herbig 1975; Snow et al. 1977; Sneden et al. 1978 ) and the photometric data taken from the 2MASS catalog (Cutri et al. 2003), we estimated the interstellar extinction of our stars to derive the intrinsic color indices $(J-H)_{0}$ and $(H-K)_{0}$. The obtained color-color diagram is shown in Fig. 4. In this figure is also shown the region occupied by Herbig Ae/Be stars (Hernández et al. 2005). We have superimposed the standard sequences of stars without emission lines having spectral types from B0 to M6 and MK luminosity classes III and V (Bessell \& Brett 1988; Glass et al. 1999). Thus, we note that classical 
T. Semaan et al.: Study of a sample of faint Be stars in the exofield of CoRoT. I.
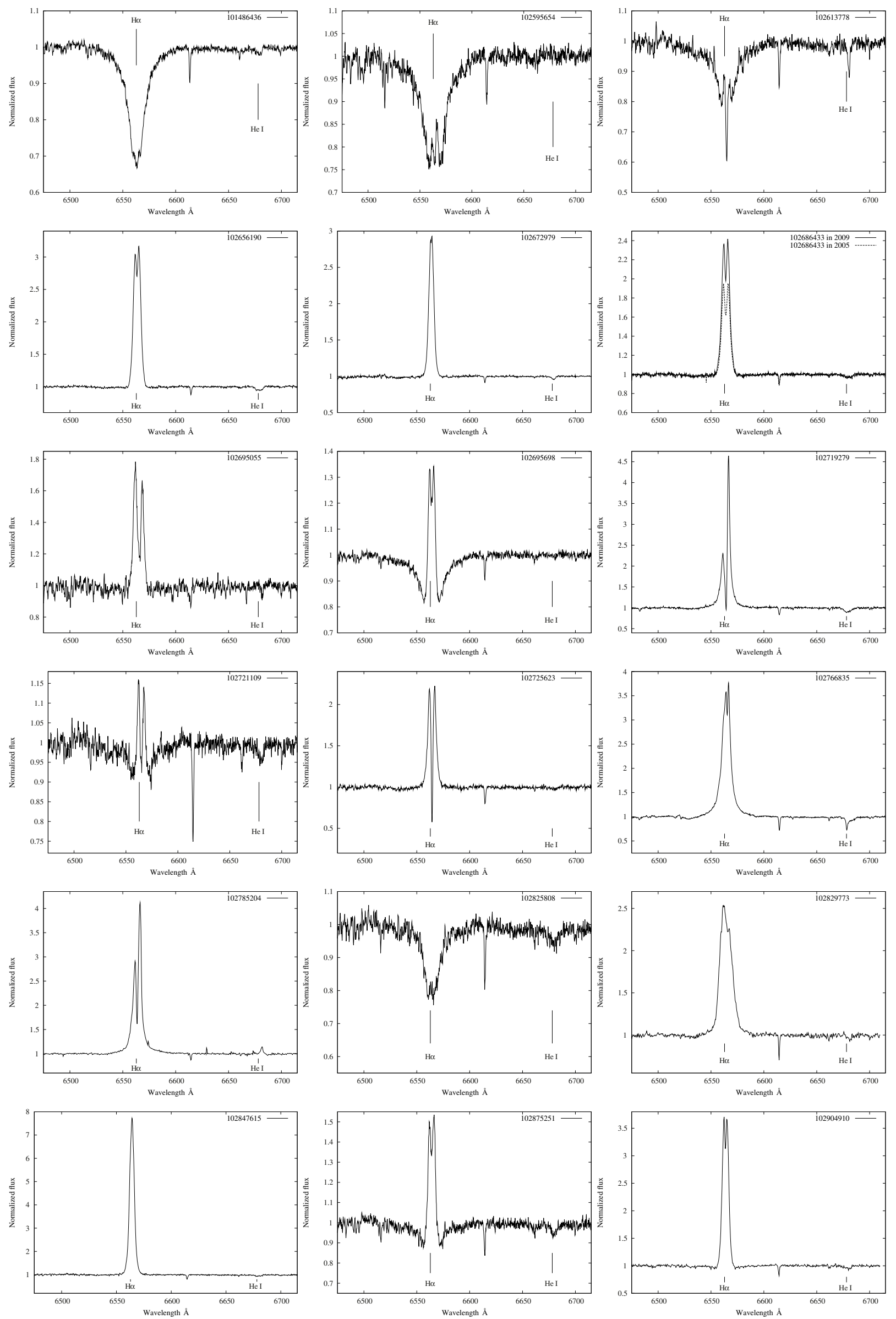

Fig. 3. $\mathrm{H} \alpha$ line profiles of the program Be stars. 
Table 3. Normalized intensity and radial velocity.

\begin{tabular}{|c|c|c|c|c|c|c|c|c|}
\hline $\begin{array}{l}\text { CoRoT ID } \\
-\end{array}$ & $\begin{array}{l}N^{*} \\
-\end{array}$ & $\begin{array}{c}\text { Line } \\
-\end{array}$ & $\begin{array}{c}I_{V} / I_{\mathrm{c}} \\
-\end{array}$ & $\begin{array}{c}R V_{v} \\
\mathrm{~km} \mathrm{~s}^{-1}\end{array}$ & $\begin{array}{c}I / I_{\mathrm{c}} \\
-\end{array}$ & $\begin{array}{c}R V \\
\mathrm{~km} \mathrm{~s}^{-1}\end{array}$ & $\begin{array}{c}I_{\mathrm{R}} / I_{\mathrm{c}} \\
-\end{array}$ & $\begin{array}{c}R V_{\mathrm{r}} \\
\mathrm{km} \mathrm{s}^{-1}\end{array}$ \\
\hline 101486436 & 1 & $\overline{\mathrm{H} \alpha}$ & 0.70 & -103 & 0.67 & +7 & 0.70 & +126 \\
\hline 102595654 & 2 & $\mathrm{H} \alpha$ & 0.81 & -42 & - & - & 0.88 & +162 \\
\hline 102613778 & 3 & $\mathrm{H} \alpha$ & 0.88 & -19 & 0.61 & $+92(\mathrm{~S})$ & 0.86 & +215 \\
\hline \multirow{2}{*}{102656190} & \multirow{2}{*}{4} & $\mathrm{H} \alpha$ & 3.0 & -45 & 2.7 & +26 & 3.2 & +106 \\
\hline & & $\mathrm{H} \gamma$ & 0.75 & -130 & 0.69 & +20 & 0.76 & +235 \\
\hline \multirow{2}{*}{102672979} & \multirow{2}{*}{5} & $\mathrm{H} \alpha$ & - & - & 2.9 & +45 & - & - \\
\hline & & $\mathrm{H} \gamma$ & 0.66 & -76 & 0.62 & +41 & 0.66 & +173 \\
\hline \multirow{2}{*}{102686433} & \multirow[b]{2}{*}{6} & $\mathrm{H} \alpha(1)$ & 1.9 & -41 & 1.6 & +54 & 1.9 & +154 \\
\hline & & $\mathrm{H} \alpha(2)$ & 2.4 & -32 & 2.0 & +52 & 2.4 & +137 \\
\hline 102695698 & 8 & $\mathrm{H} \alpha$ & 1.3 & -29 & 1.2 & +57 & 1.3 & +144 \\
\hline \multirow{2}{*}{102719279} & \multirow{2}{*}{9} & $\overline{\mathrm{H} \alpha}$ & 2.3 & -73 & 0.95 & $+70(\mathrm{~S})$ & 4.6 & +172 \\
\hline & & $\mathrm{H} \gamma$ & - & - & 0.1 & $+30(\mathrm{~S})$ & 0.9 & +168 \\
\hline 102721109 & 10 & $\mathrm{H} \alpha$ & 1.2 & -28 & 0.95 & +85 & 1.1 & +211 \\
\hline \multirow{2}{*}{102725623} & \multirow[b]{2}{*}{11} & $\mathrm{H} \alpha$ & 2.2 & -45 & 0.58 & $+75(\mathrm{~S})$ & 2.2 & +200 \\
\hline & & $\mathrm{H} \gamma$ & - & - & 0.36 & $+75(\mathrm{~S})$ & - & - \\
\hline 102728404 & 12 & $\mathrm{H} \gamma$ & - & - & 1.3 & +36 & - & - \\
\hline \multirow{2}{*}{102766835} & \multirow{2}{*}{14} & $\overline{\mathrm{H} \alpha}$ & 3.6 & +58 & 3.1 & +118 & 3.8 & +169 \\
\hline & & $\mathrm{H} \gamma$ & - & - & 0.40 & $+7(\mathrm{~S})$ & 0.95 & +160 \\
\hline \multirow{4}{*}{102785204} & \multirow{4}{*}{16} & $\mathrm{H} \gamma(1)$ & - & - & 1.2 & -36 & - & - \\
\hline & & $\mathrm{H} \alpha$ & 2.9 & -58 & 1.6 & $+33(\mathrm{~S})$ & 4.1 & +155 \\
\hline & & $\mathrm{H} \beta$ & 1.3 & -52 & 0.93 & $+35(\mathrm{~S})$ & 1.7 & +171 \\
\hline & & $\mathrm{H} \gamma(3)$ & 0.97 & -52 & 0.84 & $+34(\mathrm{~S})$ & 1.2 & +174 \\
\hline 102785480 & 17 & $\mathrm{H} \gamma$ & - & - & - & - & - & - \\
\hline 102825808 & 18 & $\mathrm{H} \alpha$ & - & - & 0.81 & +40 & - & - \\
\hline \multirow{3}{*}{102829773} & \multirow{3}{*}{19} & $\mathrm{H} \alpha$ & - & - & 2.5 & -34 & - & - \\
\hline & & $\mathrm{H} \beta$ & 1.2 & -117 & 0.98 & +120 & 1.1 & +264 \\
\hline & & $\mathrm{H} \gamma$ & 0.9 & -117 & 0.77 & +81 & - & - \\
\hline \multirow{2}{*}{102847615} & \multirow{2}{*}{20} & $\mathrm{H} \alpha$ & - & - & 7.7 & +62 & - & - \\
\hline & & $\mathrm{H} \gamma$ & 0.80 & -10 & 0.75 & +51 & 0.80 & +113 \\
\hline 102875251 & 21 & $\mathrm{H} \alpha$ & 1.5 & -30 & 1.3 & +58 & 1.5 & +153 \\
\hline \multirow{3}{*}{102904910} & \multirow{3}{*}{22} & $\overline{\mathrm{H} \alpha}$ & 3.7 & -23 & 3.1 & $+35(\mathrm{~S})$ & 3.7 & +102 \\
\hline & & $\mathrm{H} \beta$ & 1.1 & -54 & 0.93 & $+42(S)$ & 1.1 & +137 \\
\hline & & $\mathrm{H} \gamma$ & 0.74 & -98 & 0.67 & $+51(\mathrm{~S})$ & 0.74 & +264 \\
\hline
\end{tabular}

Notes. (1) Indicates the value in 2005, (2) the values in 2009, and (3) the value in 2010.

Be stars are not only separated well from the Herbig Ae/Be region, but are also situated clearly below the sequence of stars without emission lines.

Three stars, ID 102672979, ID 102728404, and ID 102785204, have much stronger infrared excesses than the remaining Be stars studied here, which possibly means that they may have wider and denser gaseous circumstellar disks. These Be stars are, however, below the region of Herbig Ae/Be stars. The location of ID 102695055, which is placed at the dwarf K5-type stars locus in the sequence, implies that the star could be a cool star with emission lines.

\section{Fundamental parameter determination}

Our aim is to obtain stellar fundamental parameters that can help us obtain reliable estimates of masses, ages, and radii of the Be stars observed by CoRoT, which are rapid rotators. These quantities will help us compare their pulsation-instability properties among them and with those predicted by the theory. They will also enable an estimate of their intrinsic rotational frequency. The determination of the fundamental parameters implies several steps:

1. first, we correct the spectra of stars with emission lines from the "veiling" effect induced by the presence of the circumstellar-envelope/disk (hereafter called CE);
2. the veiling-corrected spectra are then used to determine the "apparent" fundamental parameters. They are estimated with plane-parallel models of stellar atmospheres. These parameters summarize the spectral characteristics in a shortwavelength interval produced by the observed stellar hemisphere, which is actually deformed rotationally and has nonuniform surface temperature and gravity;

3. finally, using models that take the effects of rapid rotation into account, stellar geometrical deformation and "gravity darkening", the adopted apparent quantities are translated into "true" fundamental stellar parameters. By "true" parameters we mean those parameters corresponding to the nonrotating stellar counterpart.This mainly refers to those quantities that lead us to estimate the true stellar mass and evolutionary stage. Among "true" parameters we also mean here the equatorial radius of the rotating star and its $v \sin i$ corrected for the rotationally induced underestimation.

We could determine the fundamental parameters for 14 Be stars.

\subsection{Correction of spectra for the veiling effect}

The apparent parameters are determined using the spectra observed with FLAMES/GIRAFFE and X-shooter at ESO (LR2 spectra and UVB spectra) in the 4000-4500 $\AA$ wavelength interval, normalized to the continuum spectrum. The 


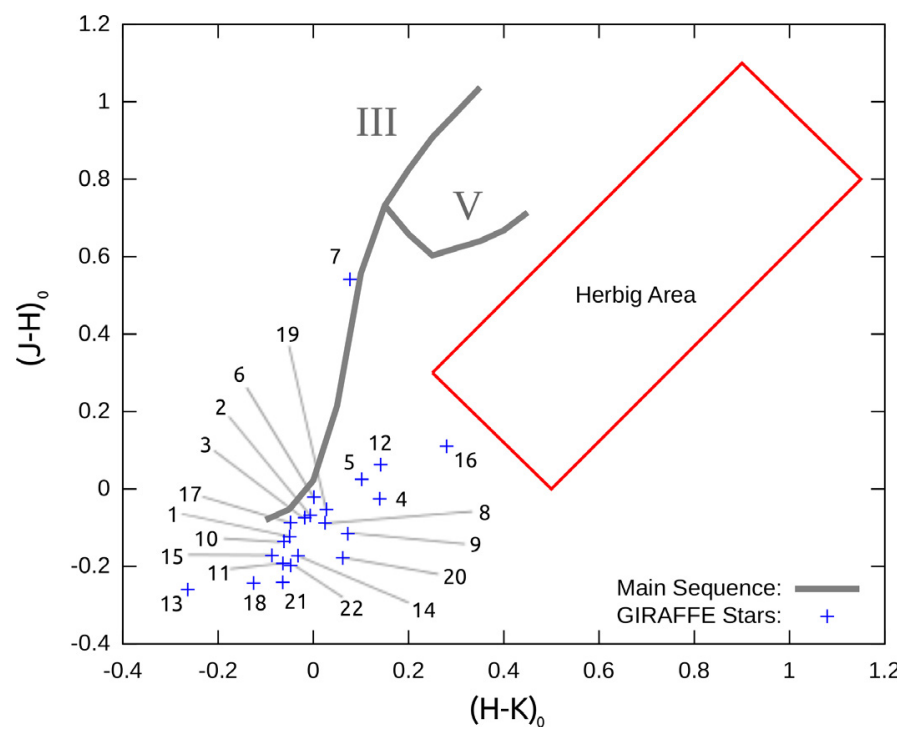

Fig. 4. JHK color-color diagram based on 2MASS magnitudes corrected from interstellar extinction of Be stars observed in the first CoRoT runs. The box shows the area of Herbig HAe/Be stars. This diagram also shows the standard sequences of intrinsic colors of stars without emission lines having luminosity classes III and V. Both sequences overlap in the $(J-K)_{\mathrm{o}}<0.7 \mathrm{mag}$ region (Glass et al. 1999). The Be stars are identified with the series number $N^{*}$ given in Table 2.

normalizing continuum has, however, two components: a stellar and a circumstellar one, which can represent a significant fraction of the total flux. The residual intensities in the photospheric components of spectral line profiles, useful for inferring the fundamental stellar parameters, can thus be considerably different from what they are expected to be if they were derived from the observed stellar spectrum normalized with a continuum that is not affected by emission and absorptions produced in the CE.

The lessening of the residual intensity in photospheric lines produced by the continuum radiation coming from the $\mathrm{CE}$ in emission-line stars and known since the fifties, was called "veiling effect" by de Jager \& Neven (1959), and first described theoretically by Hawkins (1961). This effect was extensively considered in studies of $\mathrm{T}$ Tauri stars in the eighties. For the empirical treatment of this effect, the following basic expression is used (Hartigan et al. 1989):

$\Phi^{\mathrm{obs}}(\lambda)=k[S(\lambda)+V(\lambda)]$,

where $\Phi^{\mathrm{obs}}(\lambda)$ is the observed spectrum; $S(\lambda)$ is the spectrum of a standard star, i.e. the underlying photospheric, reference or comparison spectrum; $V(\lambda)$ is the veiling spectrum; and $k$ is a normalization constant. When Eq. (1) is considered in successive limited wavelength intervals, in each of them it represents the equation of a straight line, where $k$ and $V$ are the unknown constants. Because $\Phi^{\mathrm{obs}}(\lambda)$ and $S(\lambda)$ are affected by errors, the values of $k$ and $V$ are determined using the corresponding $\chi^{2}$ test applied to Eq. (1) (Meyer 1975), which transforms the formal aspect of Eq. (1) into those currently found in the literature, as in (Hartigan et al. 1989) or Chelli (1999), but which basically refers to the same spectral elements $S(\lambda)$ and $V(\lambda)$ given in Eq. (1).

Contrary to common practice for T Tauri stars, where $S(\lambda)$ is the spectrum of a normal standard star, in Be stars this is affected by the absorption produced by the CE. Circumventing calculations of radiation transfer effects, the presence of the CE can be taken into account by using known first-order expressions that describe the line+continuum spectrum $F^{\mathrm{obs}}(\lambda)$ of the star+CE system and only the continuum spectrum $F_{\mathrm{c}}^{\mathrm{obs}}(\lambda)$ alone of the same star+CE system, which are respectively given by

$$
\begin{aligned}
& F^{\mathrm{obs}}(\lambda)=F^{*}(\lambda) A(\lambda)+E(\lambda), \\
& F_{\mathrm{c}}^{\mathrm{obs}}(\lambda)=F_{\mathrm{c}}^{*}(\lambda) A_{\mathrm{c}}(\lambda)+E_{\mathrm{c}}(\lambda),
\end{aligned}
$$

where $F^{*}(\lambda)$ and $F_{\mathrm{c}}^{*}(\lambda)$ are respectively the total and the continuum alone photospheric radiation fluxes, $A(\lambda)$ and $A_{\mathrm{c}}(\lambda)$ represent respectively the total and the continuum alone absorptions in the CE, $E(\lambda)$, and $E_{\mathrm{c}}(\lambda)$ are the total and the continuum $\mathrm{CE}$ emission terms, respectively. The observed and photospheric energy spectra are then given by $\Phi^{\mathrm{obs}}(\lambda)=F^{\mathrm{obs}}(\lambda) / F_{\mathrm{c}}^{\mathrm{obs}}(\lambda)$ and $\Phi^{*}(\lambda)=F^{*}(\lambda) / F_{\mathrm{c}}^{*}(\lambda)$, respectively.

As defined in Eq. (1), the amount of the veiling effect is only calculated from the residual intensities in the wavelengths of photospheric lines, which are not supposed to be marred by circumstellar line emissions and line self-absorptions in the CE. Hydrogen lines with emission components and other low ionization metallic transitions with emissions, are thus avoided. We write the total optical depth in the $\mathrm{CE}$ for the wavelengths in a given spectral line as $\tau_{\lambda}=\tau_{\Lambda}+\tau_{\mathrm{c}}$, where $\tau_{\Lambda}$ corresponds to the absorption inside the spectral line and $\tau_{\mathrm{c}}$ to the continuum bound-free, free-free, and electron scattering in the same wavelengths. The condition that the $\mathrm{CE}$ is optically thin in the wavelengths of certain photospheric spectral lines requires that in those wavelengths it is $\tau_{\lambda}=\tau_{\mathrm{c}}$, which carries $A(\lambda)=A_{\mathrm{c}}(\lambda)$ and $E(\lambda)=E_{\mathrm{c}}(\lambda)$ in Eq. (2). Using Eq. (2), the revised expression for the veiling effect in the mentioned " $\mathrm{CE}$ emission- and absorption-free" spectral lines, therefore becomes

$\Phi^{\mathrm{obs}}(\lambda)=\frac{\Phi^{*}(\lambda)+r(\lambda)}{1+r(\lambda)}$,

where

$r(\lambda)=\frac{E_{\mathrm{c}}(\lambda)}{F_{\mathrm{c}}^{*}(\lambda) A_{\mathrm{c}}(\lambda)}$

is called the "veiling factor". Equation (3) has exactly the same form as the one used by Basri \& Batalha (1990) and their collaborators in many other papers dealing with T Tauri stars during the nineties. While in Basri \& Batalha (1990) the veiling term is denoted $V$, we conserve the notation $r$, because we are among those few that used the concept of veiling for Be stars (Hubert-Delplace et al. 1982; Ballereau et al. 1995) and produced an empirical relation between $r$ and the emission intensity in the $\mathrm{H} \gamma$ line, published in Ballereau et al. (1995). For T Tauri stars is currently assumed that $A_{\mathrm{c}}=1$. We note that in the present work we do not tray to disentangle the values of $E_{\mathrm{c}}(\lambda)$ and $A_{\mathrm{c}}(\lambda)$ entering Eq. (4).

The effect of the veiling on the photospheric lines can be appreciated using line equivalent widths. By definition, the equivalent width of a line is $E W_{\lambda}=\int_{-\infty}^{\infty}[1-\Phi(\lambda)] \mathrm{d} \lambda$, so that

$E W_{\lambda}^{*}=(1+r) \times E W_{\lambda}^{\mathrm{obs}}$

measures the relation between the actual equivalent width of the line $\mathrm{EW}_{\lambda}^{*}$ and the apparent one $\mathrm{EW}_{\lambda}^{\text {obs }}$ derived from the veiled spectrum. We note that in the wavelengths of the continuum, i.e. where $\Phi^{\mathrm{obs}}(\lambda)=1$, Eq. (3) produces $\Phi^{*}(\lambda)=1$ regardless of the value of $r$, as expected.

For an order of magnitude estimate of $r$ in the surroundings of the $\mathrm{H} \gamma$ line, we consider a typical Be star with $T_{\text {eff }} \simeq$ $22000 \mathrm{~K}$, an average CE temperature $T_{\text {env }} \simeq 0.5 \times T_{\text {eff }}$, effective radius of the continuum energy emission region $R_{\mathrm{e}} / R_{*} \simeq 1.2$, and $\tau_{\mathrm{c}} \simeq 0.5$ (Moujtahid et al. 2000b,a). Using expressions for 
Table 4. Adopted average veiling factors $\langle 1+r\rangle$ to correct the energy distributions of our program Be stars.

\begin{tabular}{lcccc}
\hline \hline CoRoT ID & $N^{*}$ & $E W_{\mathrm{H} \gamma}(\AA)$ & $I_{\mathrm{H} \gamma}(\AA)$ & $\langle 1+r\rangle$ \\
- & - & $\AA$ & $\AA$ & - \\
\hline 101486436 & 1 & -0.30 & 0.094 & $0.93 \pm 0.01$ \\
102595654 & 2 & -0.90 & 0.186 & $1.08 \pm 0.10$ \\
102656190 & 4 & $-0,85$ & 0.611 & $1.13 \pm 0.05$ \\
102672979 & 5 & -0.49 & 0.218 & $0.99 \pm 0.02$ \\
102686433 & 6 & -0.55 & 0.254 & $1.01 \pm 0.02(1)$ \\
102719279 & 9 & -0.67 & 0.298 & $1.05 \pm 0.03(2)$ \\
102725623 & 11 & -0.75 & 0.615 & $1.11 \pm 0.01$ \\
102728404 & 12 & -2.23 & 0.235 & $1.04 \pm 0.05$ \\
102766835 & 14 & -1.12 & 2.238 & $1.69 \pm 0.06$ \\
102785480 & 17 & +0.23 & -0.165 & $1.45 \pm 0.18$ \\
102825808 & 18 & -0.10 & 0.062 & $0.89 \pm 0.10$ \\
102829773 & 19 & -1.46 & 1.401 & $1.39 \pm 0.05$ \\
102847615 & 20 & -1.27 & 0.721 & $1.24 \pm 0.10$ \\
102904910 & 22 & -0.78 & 0.499 & $1.10 \pm 0.03$ \\
\hline
\end{tabular}

Notes. (1) Indicates the value in 2005 and (2) the value in 2009.

$A_{\mathrm{c}}$ and $E_{\mathrm{c}}$ given in Moujtahid et al. (1999) or Moujtahid et al. (2000a), we obtain $r \simeq 0.4$. The rather high value of $r$ thus inferred suggests that the fit of uncorrected energy distributions with model atmospheres may easily lead to misleading fundamental parameter determinations (Frémat et al. 2006).

In Eq. (4), the veiling factor is explicitly considered as a function of $\lambda$. Taking the expressions in Moujtahid et al. (1999, 2000a) for the terms entering Eq. (4), it appears that the value of $r$ increases in $\Delta r / r \lesssim 0.03$ from $\lambda=4000 \AA$ to $\lambda=4500 \AA$. Because this difference is smaller than the uncertainty related with the empirical estimate of $r$ at $\lambda=4340 \AA$ from the calibrations of $r$, we take $r=$ constant over the $\lambda \lambda$ 4000-4500 wavelength interval.

As noted above, to correct the observed spectra of our program Be stars for the veiling effect, we used the correlation between $r$ and the emission component in the $\mathrm{H} \gamma$ line obtained for Be stars by Ballereau et al. (1995). These authors have observed that the CE emission in the continuum becomes sensitive once the emission in the $\mathrm{H} \gamma$ line becomes measurable. The regression lines between $r$ and the equivalent width $\mathrm{EW}_{\mathrm{H} \gamma}$, and with the intensity $I_{\mathrm{H} \gamma}$ of the emission component in the $\mathrm{H} \gamma$ line, are given by

$$
\begin{aligned}
& 1+r=\left(-E W_{\mathrm{H} \gamma}+1.87\right) / 2.35, \quad \text { EW in } \AA \\
& 1+r=\left(I_{\mathrm{H} \gamma}+2.84\right) / 3.12 .
\end{aligned}
$$

The intensity of the emission, normalized to the surrounding continuum, is defined as

$I_{\mathrm{H} \gamma}=-E W_{\mathrm{H} \gamma}\left[\frac{F_{\mathrm{H} \gamma}^{\mathrm{c}}}{F_{\mathrm{H} \gamma}^{\mathrm{c}}(22500,4.0)}\right]$,

where $F_{\mathrm{H} \gamma}^{\mathrm{c}}$ is the continuum flux at the $\mathrm{H} \gamma$ line, and $F_{\mathrm{H} \gamma}^{\mathrm{c}}(22500,4.0)$ is the continuum flux at the same wavelengths given by a model of stellar atmosphere for $T_{\text {eff }}=22500 \mathrm{~K}$ and $\log g=4.0$. Table 4 reproduces the estimated $r$ veiling factors of our program Be stars.

\subsection{Apparent fundamental parameters}

The apparent parameters do not take the effects induced by a rapid stellar rotation into account, i.e. stellar flattening and the concomitant gravitational darkening, but simply represent the spectrum from 4000 to $4500 \AA$ A emitted by an equivalent spherical star with uniform temperature and surface gravity.

To derive the apparent fundamental parameters, planeparallel model atmospheres were computed for $T_{\text {eff }}$ ranging from $8000 \mathrm{~K}$ to $55000 \mathrm{~K}$ and $\log g$ ranging from 2.5 to $4.5 \mathrm{dex}$ in two steps. First, the temperature structure of the atmospheres was computed with the ATLAS9 FORTRAN program (Kurucz 1993; Castelli et al. 1997). For $15000 \mathrm{~K}<T_{\text {eff }}<27000 \mathrm{~K}$, non-LTE level populations were calculated for the considered atoms using TLUSTY (Hubeny \& Lanz 1995) by keeping the temperature and density distributions fixed, and we adopted the solar chemical abundances given by Grevesse \& Sauval (1998). For model atmospheres with effective temperatures lower than $T_{\text {eff }}<15000 \mathrm{~K}$, full LTE was assumed, while those with $T_{\text {eff }}>27000 \mathrm{~K}$ are models from the OSTAR2002 non-LTE grid (Lanz \& Hubeny 2003). The grid of fluxes we use during the fitting procedure (Sect. 4.1) was built with SYNSPEC. Other models computing details are given in Frémat et al. (2005, 2006).

The apparent effective temperature and surface gravity are based on the fit of hydrogen line wavelengths not affected by the CE emission/absorption, but mostly helium, carbon, oxygen, silicon, and $\mathrm{Mg}$ II lines in the spectral domain ranging from 4000 to $4500 \AA$. The normalized spectra of B and Be stars are Dopplershifted according to the radial velocity. They are also corrected for the veiling effect; i.e., we derive the spectrum $\Phi^{*}(\lambda)$ with Eq. (3) in Sect. 6.1 using $\Phi^{\mathrm{obs}}(\lambda)$ and the corresponding veiling factor $r$ given in Table 4 .

The veiling-corrected spectra were compared to a grid of synthetic spectra using a robust least squares method in the MINUIT minimization package developed at CERN. This package was transcribed into a FORTRAN language and named GIRFIT (Frémat et al. 2006). The synthetic spectra were convolved with a Gaussian function for the required instrumental resolution. To determine the $v \sin i$ of the studied stars, the spectral lines were Doppler-broadened for several apparent rigid rotational velocities. In each case the fit was controlled with a $\chi^{2}$ test computed over specific zones in the 4000 to $4500 \AA$ wavelength range, chosen so as to exclude spectral characteristics affected by line emission and/or shell absorption (e.g. hydrogen line cores). These zones are also free from interstellar absorption bands, generally found in spectra of distant stars.

An example of the best fit of observed spectra with GIRFIT, uncorrected and corrected for the veiling effect, is shown in Fig. 5. The spectral adjustments obtained for the other stars are shown in the electronic version of this paper. The derived apparent fundamental parameters corrected for veiling when needed are given in Table 5. The uncertainties on the determination of the apparent effective temperature are $\epsilon_{T_{\text {eff }}} \simeq 0.07 \times T_{\text {eff }}$ on average, while they are $\epsilon_{\log g} \simeq 0.3$ dex on the apparent surface gravity.

\subsection{Correction of fundamental parameters for rotational effects}

In this section, we assume that stars are rigid rotators characterized by a surface rate of angular velocity $\omega=\Omega / \Omega_{\mathrm{c}}$, where $\Omega_{\mathrm{c}}$ is the surface angular velocity at critical rotation. The 
T. Semaan et al.: Study of a sample of faint Be stars in the exofield of CoRoT. I.
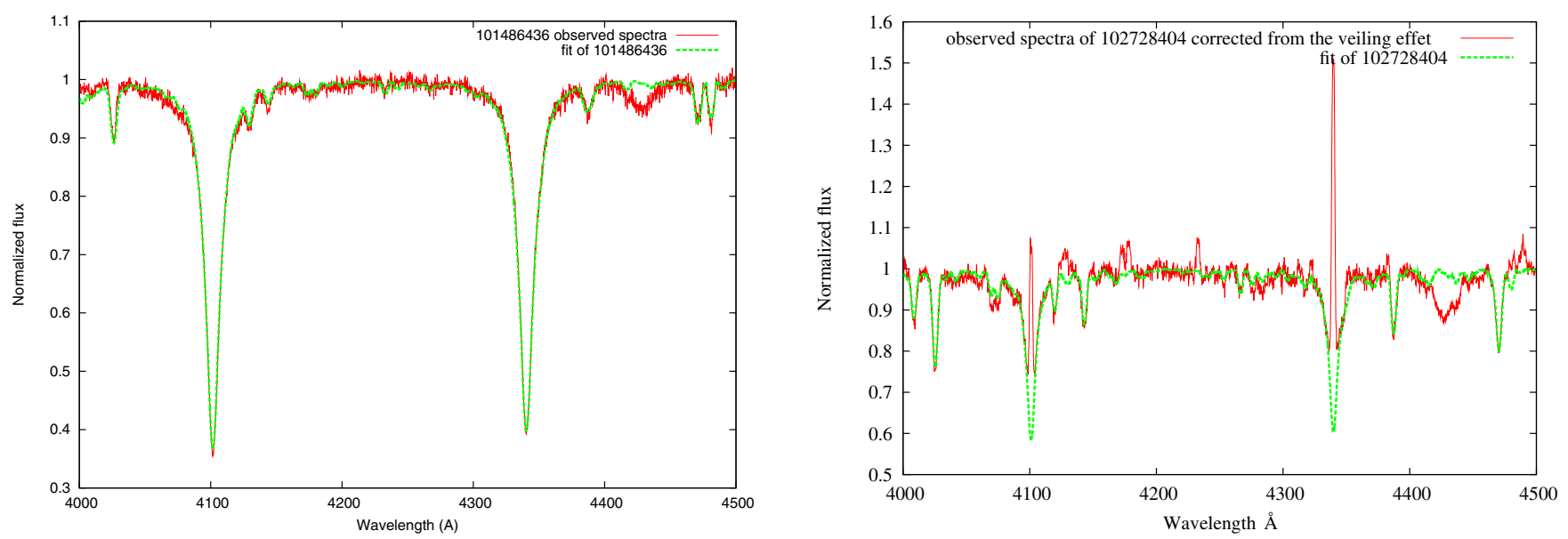

Fig. 5. Left: fit of the observed spectrum of the star CoRoT ID 101486436 with GIRFIT in the wavelength interval $\lambda \lambda 4000-4500 \AA$ A. Right: fit of the observed spectrum corrected from the veiling effects of the star CoRoT ID 102728404 in the same wavelength interval.

Table 5. Apparent fundamental parameters.

\begin{tabular}{cccccc}
\hline \hline \multirow{2}{*}{ CoRoT ID } & $N^{*}$ & $\begin{array}{c}T_{\text {eff }} \pm \epsilon_{T_{\text {eff }}} \\
\mathrm{K}\end{array}$ & $\begin{array}{c}\log g \pm \epsilon_{\log g} \\
\mathrm{dex}\end{array}$ & $\begin{array}{c}v \sin i \pm \epsilon_{v \sin i} \\
\mathrm{~km} \mathrm{~s}^{-1}\end{array}$ & $\begin{array}{c}\log L \pm \epsilon_{\log L} \\
L_{\odot}\end{array}$ \\
\hline 101486436 & 1 & $12000 \pm 1500$ & $4.0 \pm 0.2$ & $220 \pm 30$ & $2.24 \pm 0.26$ \\
102595654 & 2 & $10000 \pm 1500$ & $3.3 \pm 0.3$ & $330 \pm 30$ & $2.67 \pm 0.27$ \\
102656190 & 4 & $19000 \pm 2000$ & $4.0 \pm 0.2$ & $260 \pm 30$ & $3.33 \pm 0.26$ \\
102672979 & 5 & $14500 \pm 2000$ & $3.3 \pm 0.4$ & $200 \pm 30$ & $3.55 \pm 0.28$ \\
102686433 & 6 & $14750 \pm 1500$ & $3.4 \pm 0.2$ & $275 \pm 30$ & $3.46 \pm 0.27$ \\
102719279 & 9 & $20000 \pm 1500$ & $3.5 \pm 0.2$ & $270 \pm 20$ & $4.08 \pm 0.28$ \\
102725623 & 11 & $12500 \pm 1500$ & $2.7 \pm 0.3$ & $300 \pm 40$ & $4.02 \pm 0.30$ \\
102728404 & 12 & $22500 \pm 2000$ & $3.9 \pm 0.3$ & $190 \pm 30$ & $3.87 \pm 0.27$ \\
102766835 & 14 & $32000 \pm 2000$ & $3.7 \pm 0.3$ & $250 \pm 50$ & $5.11 \pm 0.33$ \\
102785480 & 17 & $19000 \pm 2000$ & $3.6 \pm 0.2$ & $310 \pm 30$ & $3.82 \pm 0.27$ \\
102825808 & 18 & $17500 \pm 2000$ & $3.8 \pm 0.3$ & $340 \pm 40$ & $3.37 \pm 0.26$ \\
102829773 & 19 & $21015 \pm 2000$ & $4.2 \pm 0.3$ & $390 \pm 40$ & $3.34 \pm 0.26$ \\
102847615 & 20 & $16500 \pm 2000$ & $4.1 \pm 0.3$ & $200 \pm 30$ & $2.87 \pm 0.26$ \\
102904910 & 22 & $17000 \pm 2000$ & $3.9 \pm 0.2$ & $270 \pm 30$ & $3.18 \pm 0.26$ \\
\hline
\end{tabular}

apparent fundamental parameters obtained in Sect. 6.2 can then be given with the following formal aspect:

$$
\begin{aligned}
T_{\text {eff }}^{\text {app }} & =\left\langle T_{\text {eff }}\right\rangle \times f_{\mathrm{T}}(M, t, i, \omega), \\
\log g^{\text {app }} & =\log \left[\langle g\rangle \times f_{\mathrm{G}}(M, t, i, \omega)\right], \\
L^{\text {app }} & =\langle L\rangle \times f_{\mathrm{L}}(M, t, i, \omega), \\
(v \sin i)_{\text {true }} & =V_{\mathrm{c}}(M, t)\left[\frac{R_{\mathrm{eq}}(M, t, \omega)}{R_{\mathrm{c}}(M, t)}\right] \sin i,
\end{aligned}
$$

where $M$ is the true stellar mass, $t$ the stellar age, $i$ the inclination angle of the rotation axis, $V_{\mathrm{c}}$ the linear critical rotational velocity at the equator, $R_{\mathrm{eq}}$ the stellar true equatorial radius, and $R_{\mathrm{c}}$ the stellar true equatorial radius at critical rigid rotation. The parameters marked as $\langle\ldots\rangle$ are average values over the entire geometrically deformed surface of the rotating star. The factors $f_{\mathrm{T}}$ and $f_{\mathrm{G}}$ are calculated with FASTROT as follows. For the required stellar masses, ages, and rotational rates, we calculated 2D models of stellar structure assuming rigid rotation (Zorec et al. 2011; Zorec \& Royer 2012). The obtained rotationally induced stellar geometrical deformations with the surface gravities depending on the stellar latitude. We calculated the surface effective temperature distribution using the classical von Zeipel theorem (von Zeipel 1924). With local plane-parallel model atmospheres, we recomposed the apparent stellar spectra in the $\lambda \lambda 4000-4500 \AA$ wavelength region as observed at several inclination angles $i$.

To simplify the calculations, in the present work the functions $f_{\mathrm{T}}$ and $f_{\mathrm{G}}$ were obtained with respect to the quantities $\left\langle T_{\mathrm{eff}}\right\rangle$ and $\langle g\rangle$ averaged over the rotationally deformed stellar surface for the given $\omega$ rates. They have, however, the same aspect as the similar ones given by Frémat et al. (2005, their Fig. 2), which were calculated with respect to $T_{\mathrm{o}}$ and $g_{\mathrm{o}}$, respectively as the effective temperature and gravity of the parent nonrotating stellar counterparts.

Solutions of systems of equations similar to Eq. (8) have been done in Floquet et al. (2000), Neiner et al. (2003), Frémat et al. (2005), Zorec et al. (2005), and Vinicius et al. (2006). The observed quantities are on the lefthand side of Eq. (8), except for $v \sin i$ for which we have written its true value. In fact, due to gravitational darkening, the equatorial regions have lower local effective temperatures, which then contribute less efficiently to the Doppler-broadening of the spectral line. The observed (apparent) $v \sin i$ can then be underestimated in rapid 
rotators (Stoeckley 1968; Townsend et al. 2004; Frémat et al. 2005). The amount of this underestimation was computed by Frémat et al. (2005) and is automatically taken into account in the interpolations that solve the system of simultaneous equations in Eq. (8). The evolutionary tracks calculated by Meynet \& Maeder (2000), and Ekström et al. $(2008,2012)$ are given in terms of effective temperatures and bolometric luminosities averaged over the surface of geometrically deformed rotating stars. The system of equations Eq. (8) is solved by iteration, where the initial masses and ages are interpolated in evolutionary tracks without rotation (Ekström et al. 2012) using the apparent effective temperatures and bolometric luminosities. At each iteration step we determine the rotational velocity of stars in the ZAMS and interpolate then the stellar mass and age using the evolutionary tracks with rotation that are consistent with the obtained initial rotational rates.

We note, however, that in the present case we only have three apparent parameters obtained with the GIRFIT fitting procedure, while Eq. (8) is a system of four equations. We then parameterized the rotation rate $\omega=\Omega / \Omega_{\mathrm{c}}$ as previously done in Frémat et al. (2006) and Martayan et al. (2006, 2007) and obtained for each case the quantities such as those given in Table 6 for $\Omega / \Omega_{\mathrm{c}}=0.95$ used as an example. We assume that the errors on the $\left\langle T_{\text {eff }}\right\rangle$ and $\log \langle g\rangle$ are similar to those on their apparent counterparts. When the parameters $\left\langle T_{\text {eff }}\right\rangle$ and $\log \langle g\rangle$ are used with their uncertainties to determine the bolometric luminosities from the evolutionary tracks, the average $\left\langle\log L / L_{\odot}\right\rangle$ is characterized by the $\epsilon_{\langle\log L\rangle}$ uncertainties (or $1 \sigma$ ) reported in Table 6 . This table also gives the rotation frequencies calculated as

$v_{\mathrm{r}}=0.2\left[\frac{V_{\mathrm{eq}}(M, t, \omega)}{R_{\mathrm{eq}}(M, t, \omega)}\right] \quad$ cycles $/$ day,

where $V_{\mathrm{eq}}$ is the true equatorial linear rotational velocity (in $\mathrm{km} \mathrm{s}^{-1}$ ) and $R_{\mathrm{eq}}$ is, in solar units, the equatorial radius of the rotationally distorted star. Table 6 also gives the uncertainties affecting the estimate of $v_{\mathrm{r}}$. They are estimated using the uncertainties on the estimates of $v \sin i, R_{\mathrm{eq}}$, and on the inclination $i$. In Table 6 are also given the fractional ages of stars $\tau / \tau_{\mathrm{MS}}$, where $\tau$ is the age of the star and $\tau_{\text {MS }}$ the time a star of a given mass remains in the main sequence evolutionary phase (MS).

Finally we transcribed in Table 6 the fundamental parameters we obtained into spectral type and luminosity classes thanks to effective temperature and surface gravity calibration (Gray \& Corbally 1994; Zorec 1986) as used in Martayan et al. (2006).

\subsection{The apparent and true HR diagram of the program Be stars}

In Fig. 6 the positions of our stars are shown as a function both of the apparent and of surface-averaged fundamental parameters calculated for $\Omega / \Omega_{\mathrm{c}}=0.95$. As in previously cited works, the "true" positions of stars correspond on average to hotter and slightly more massive stars than expected from the apparent parameters. In spite of the uncertainties on the fundamental parameters, there are two objects (CoRoT 102725623, star $N^{*}=11$ and CoRoT 102595654, star $N^{*}=2$ ) that remain beyond the MS phase. Their Be phenomenon could be thought of as favored by the rotational acceleration they acquire during the secondary contraction phase. According to Negueruela (2004) some of them could even be interacting binaries. This figure also shows the evolutionary tracks of rotating stars whose initial equatorial rotational velocities in the ZAMS are $V_{\text {ini }} / V_{\mathrm{c}}=0.4$ (Ekström et al. 2012).

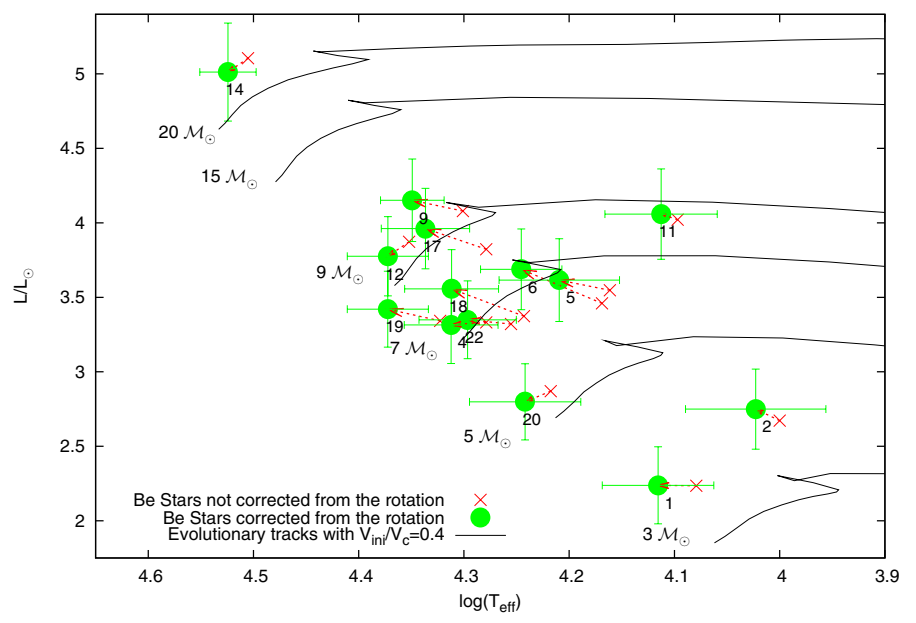

Fig. 6. HR diagram of the studied Be stars. The evolutionary tracks with rotation are from Ekström et al. (2012), and are calculated for $V_{\text {ini }} / V_{\mathrm{c}}=0.4$.

\section{Discussion}

For several Be stars, we have been able to compare the spectral classification we derived from spectroscopy with the one obtained by Gutiérrez-Soto (2006) from Strömgren photometry (see Table 7). There is good agreement for the stars 102785480 , 102656190, and 102725623, which show a weak emission in their blue spectra. For the two other stars there is no agreement: the star 102719279 shows strong He I lines that are inconsistent with a B9 spectral type, and the star 102766835 shows a strong He II 4200 line that is not expected in a B2 star. In addition, there are strong shell features in these last two objects. Then, it is not excluded that the flux excesses or flux deficiencies produced by the circumstellar disk lead to color photometric indices that do not reflect the right spectral type of the objects.

In fact, it is well known that during a phase without (or with very low) emission, a Be star shows an energy distribution around the Balmer discontinuity (BD) that resembles that of a normal-B star. Since Barbier \& Chalonge (1939), it is also known that Be stars can show two Balmer discontinuities. The first one resembles that of a normal-B star, and it has the same average spectral location. The second BD appears at shorter wavelengths than the first one, which indicates that it forms in a medium with lower gas pressure than in the photosphere of the central star. While the first BD is constant and can be used for spectral classification or apparent fundamental parameter determination, the second BD is variable, which according to the epoch can be either in emission or in absorption (Moujtahid et al. 1998). We note that the variable component of the BD enters the Strömgren photometric filter $u$ (or $U$ filter of the UBV system), which can then produce photometric indices that do not reflect the genuine photospheric BD of the star and leads to wrong spectral classifications, as well as to bad fundamental parameter determinations (Divan \& Zorec 1982). The effects of circumstellar emission in the bands and indices of the Strömgren system have been studied in detail by Fabregat \& Reglero (1990) and Fabregat et al. (1996). They clearly show that circumstellar emission or absorption significantly modify the values of the $(b-y)$ and $c_{1}$ indices, which cannot be considered as effective temperature indicators without applying the adequate corrections. The flux excesses, whether positive or negative, implied in the second BD are accompanied by changes of the visual 
T. Semaan et al.: Study of a sample of faint Be stars in the exofield of CoRoT. I.

Table 6. Fundamental parameters and rotational frequencies corrected for rotational effects assuming that $\omega=\Omega / \Omega_{\mathrm{c}}=0.95$.

\begin{tabular}{|c|c|c|c|c|c|c|c|c|c|c|}
\hline CoRoT ID & $N^{*}$ & Spec. Class. & $\begin{array}{c}\left\langle T_{\text {eff }}\right\rangle \\
\mathrm{K}\end{array}$ & $\begin{array}{c}\log \langle g\rangle \\
\operatorname{dex}\end{array}$ & $\begin{array}{c}v \sin i \\
\mathrm{~km} \mathrm{~s}^{-1}\end{array}$ & $\begin{array}{c}\log \langle L\rangle \pm \epsilon_{\log \langle L\rangle} \\
L_{\odot}\end{array}$ & $\begin{array}{c}R \pm \epsilon_{\mathrm{R}} \\
R_{\odot}\end{array}$ & $\begin{array}{c}M \pm \epsilon_{\mathrm{M}} \\
M_{\odot}\end{array}$ & $\begin{aligned} \tau / \tau_{\mathrm{MS}} & \pm \epsilon_{\tau / \tau_{\mathrm{MS}}} \\
& -\end{aligned}$ & $\begin{array}{c}v_{\mathrm{r}} \pm \epsilon_{v} \\
\mathrm{c} / \mathrm{d}\end{array}$ \\
\hline 101486436 & 1 & B7 V & 13000 & 4.1 & 242 & $2.24 \pm 0.26$ & $2.6 \pm 0.5$ & $3.5 \pm 0.2$ & $0.30 \pm 0.23$ & $2.12 \pm 0.24$ \\
\hline 102595654 & 2 & B9 III & 10500 & 3.4 & 328 & $2.75 \pm 0.27$ & $7.3 \pm 1.5$ & $3.9 \pm 0.3$ & $1.02 \pm 0.01$ & $1.28 \pm 0.27$ \\
\hline 102656190 & 4 & B2 V & 20500 & 4.1 & 278 & $3.31 \pm 0.26$ & $3.7 \pm 0.7$ & $7.0 \pm 0.4$ & $0.23 \pm 0.21$ & $1.83 \pm 0.20$ \\
\hline 102672979 & 5 & B3 IV & 16200 & 3.5 & 222 & $3.62 \pm 0.28$ & $8.4 \pm 1.8$ & $6.8 \pm 0.5$ & $1.00 \pm 0.03$ & $0.55 \pm 0.07$ \\
\hline 102686433 & 6 & B3 IV & 17600 & 3.7 & 300 & $3.69 \pm 0.27$ & $7.7 \pm 1.6$ & $7.4 \pm 0.5$ & $0.96 \pm 0.05$ & $0.73 \pm 0.09$ \\
\hline 102719279 & 9 & B1 IV & 22300 & 3.7 & 287 & $4.15 \pm 0.28$ & $8.1 \pm 1.8$ & $10.6 \pm 0.8$ & $0.88 \pm 0.07$ & $0.73 \pm 0.09$ \\
\hline 102725623 & 11 & B7 III & 13000 & 2.8 & 296 & $4.06 \pm 0.30$ & $21.8 \pm 5.1$ & $8.9 \pm 1.0$ & $1.02 \pm 0.01$ & $0.35 \pm 0.08$ \\
\hline 102728404 & 12 & B1 V & 23600 & 4.0 & 200 & $3.78 \pm 0.27$ & $4.7 \pm 1.0$ & $9.3 \pm 0.5$ & $0.45 \pm 0.21$ & $1.40 \pm 0.15$ \\
\hline 102766835 & 14 & O9 IV & 33400 & 3.8 & 262 & $5.01 \pm 0.33$ & $9.9 \pm 2.5$ & $23.1 \pm 2.8$ & $0.63 \pm 0.12$ & $0.83 \pm 0.12$ \\
\hline 102785480 & 17 & B2 IV & 21700 & 3.8 & 331 & $3.96 \pm 0.27$ & $6.9 \pm 1.5$ & $9.5 \pm 0.7$ & $0.83 \pm 0.09$ & $0.94 \pm 0.12$ \\
\hline 102825808 & 18 & B2 V & 20500 & 4.0 & 369 & $3.56 \pm 0.26$ & $4.9 \pm 1.0$ & $7.7 \pm 0.4$ & $0.63 \pm 0.17$ & $1.45 \pm 0.19$ \\
\hline 102829773 & 19 & B1 V & 23600 & 4.3 & 409 & $3.42 \pm 0.25$ & $3.1 \pm 0.6$ & $8.2 \pm 0.4$ & $0.02 \pm 0.04$ & $2.63 \pm 0.30$ \\
\hline 102847615 & 20 & B3 V & 17500 & 4.2 & 220 & $2.80 \pm 0.26$ & $2.8 \pm 0.6$ & $5.2 \pm 0.3$ & $0.11 \pm 0.13$ & $2.29 \pm 0.25$ \\
\hline 102904910 & 22 & $\mathrm{~B} 2 \mathrm{~V}$ & 18900 & 4.1 & 296 & $3.23 \pm 0.26$ & $4.0 \pm 0.8$ & $6.4 \pm 0.3$ & $0.50 \pm 0.20$ & $1.60 \pm 0.18$ \\
\hline
\end{tabular}

Table 7. Comparison between the photometric classification and the one provided with spectroscopy.

\begin{tabular}{lcc}
\hline \hline CoRoT ID & Spectroscopic class. & Photometric class. \\
\hline 102656190 & B2 V & B3 \\
102719279 & B1 IV & B9 \\
102725623 & B7 III & B6 \\
102766835 & O9 IV & B2 \\
102785480 & B2 IV & B2 \\
\hline
\end{tabular}

photometric color indices, which also have a direct incidence on the fundamental parameter determinations. If the presence of the second $\mathrm{BD}$ and color changes of photometric indices due to the $\mathrm{CE}$ are not duly taken into account, the resulting spectral classification and fundamental parameter determinations can be misleading. For these reasons we are more confident in the spectral classification produced in the present paper.

We notice that the correction for rapid rotation produces slightly higher rotation frequencies $v_{\mathrm{r}}$ than when they are obtained with uncorrected parameters. Even though the values of $V_{\text {eq }}(M, t, \omega)$ and $R_{\text {eq }}(M, t, \omega)$ can undergo significant changes with $\omega=\Omega / \Omega_{\mathrm{c}}$, the final value of $v_{\mathrm{r}}$ varies much less, which indicates that $v_{\mathrm{r}}$ is a robust quantity that can help to distinguish pulsation phenomena from spectral signatures that might otherwise be assigned to transiting spots on the stellar surface.

The masses and ages are interpolated in evolutionary tracks with rotation. However, we can see that in general the estimates of masses do not depend strongly on the effects induced by the rotation on the fundamental parameters, while ages are rather sensitive to them. The greater $\Omega / \Omega_{\mathrm{c}}$, the younger the star can appear after correcting for rotational effects. The effect on the stellar age can be estimated easily using the following relation (Meynet \& Maeder 2000):

$$
\tau(\Omega) / \tau_{\mathrm{MS}}(\Omega)=\frac{\left(\tau / \tau_{\mathrm{MS}}\right)_{\mathrm{o}}}{1+\gamma\left(M, \Omega / \Omega_{\mathrm{c}}\right)\left(\tau / \tau_{\mathrm{MS}}\right)_{\mathrm{o}}}
$$

where $\left(\tau / \tau_{\mathrm{MS}}\right)_{\mathrm{o}}$ is the fractional age deduced from rotation-less evolutionary tracks, and the value of $\gamma$ for some masses and rates $\Omega / \Omega_{\mathrm{c}}$ are given in Zorec et al. (2005).

According to these values, we can safely say that two out of 14 program stars $\left(N^{*}=2\right.$ and $\left.N^{*}=11\right)$ are well beyond the TAMS when they are considered either with or without rotation. These stars may question us on the possible mechanism responsible for their Be phenomenon. It will then be quite important to see whether their Be characteristic is due to pulsation or other phenomena. It may happen that their circumstellar disk is only a remnant from the period of the secondary contraction, produced by a noticeable increase in the surface rotation velocity that could favor the critical rotation (Negueruela 2004).

\section{Conclusions}

Thanks to the spectra obtained with the multi-object FLAMES/ GIRAFFE spectrograph and additional spectra taken with the new-generation X-shooter spectrograph, we have been able to identify spectroscopically 17 faint Be stars in the first exoplanetary fields of CoRoT and two faint stars possibly showing the Be phenomenon. We investigated the spectral characteristics of all these Be stars. We estimated the interstellar reddening of these objects using interstellar bands contained in our spectra and obtained their intrinsic color indices in the near-IR using 2MASS data. We have found that all the studied stars depicted in the $(J-H)_{0},(H-K)_{0}$ diagram are classical Be stars, except one object that is a cool star and probably a T Tauri star. However three Be stars, which have a stronger IR excess than the average one in the remaining Be stars, also show strong emission line contamination in their visual and IR spectra, but they are still below the Herbig Ae/Be domain, which is also a way to confirm their genuine Be character. For the first time, we have determined accurate fundamental parameters $\left(T_{\text {eff }}, \log g, v \sin i\right.$, $\mathrm{M}, \mathrm{L}, \tau / \tau_{\mathrm{MS}}$, rotational frequency) and their uncertainties for angular rotational velocities ranging from $\Omega / \Omega_{\mathrm{c}}=0.80$ to 1.0 for a significant sample of faint Be stars observed by CoRoT. In the determination we have considered the veiling effect produced by the circumstellar environment and the effect induced by the very rapid rotation. We investigated the positions of our stars in the HR diagram and found that two objects are beyond the MS phase. Accurate fundamental parameters are required for the analysis of the CoRoT light curves of our sample of Be stars, particularly for the search of patterns in the frequency spectrum as a function of the evolutionary status of the studied stars. This study will be the object of a subsequent contribution. 
Acknowledgements. The work of JGS is supported by the Spanish Plan Nacional de I+D+i under contract AYA2010-20982-C02-01. The work of J.F. is supported by the spanish Plan Nacional de I+D+i and FEDER under contract AYA2010-18352, and partially supported by the Generalitat Valenciana project of excellence PROMETEO/2009/064. The CAFOS spectra were obtained by graduate students of the Valencia University, within the "Calar Alto Academy" program. We are very grateful to the staff at Paranal for their assistance for observations with VLT/FLAMES. We thank an anonymous referee for the comments that helped to improve the presentation of our results. This research made use of the Simbad database and Vizier database maintained at the CDS, Strasbourg, France, and the WEBDA database, operated at the Institute for Astronomy of the University of Vienna. This publication makes use of data products from the Two Micron All Sky Survey, which is a joint project of the University of Massachusetts and the Infrared Processing and Analysis Center/California Institute of Technology, funded by the National Aeronautics and Space Administration and the National Science Foundation. The INT telescope and its service and standard program are operated on the island of $\mathrm{La}$ Palma by the Isaac Newton Group in the Spanish Observatorio del Roque de los Muchachos of the Instituto de Astrofísica de Canarias. The Albireo spectrograph at the $1.5 \mathrm{~m}$ telescope is operated by the Instituto de Astrofísica de Andalucía at the Sierra Nevada Observatory. The CAFOS spectrograph at the $2.2 \mathrm{~m}$ telescope is operated at the German-Spanish Astronomical Center, Calar Alto, jointly operated by the Max-Planck-Institut für Astronomie Heidelberg and the Instituto de Astrofísica de Andalucía (CSIC). We warmly thank Mrs J. Adams for the language editing of this paper.

\section{References}

Baglin, A., Auvergne, M., Barge, P., et al. 2006, in ESA SP 1036, eds. M. Fridlund, A. Baglin, J. Lochard, \& L. Conroy, 33

Ballereau, D., Chauville, J., \& Zorec, J. 1995, A\&AS, 111, 423

Barbier, D., \& Chalonge, D. 1939, ApJ, 90, 627

Basri, G., \& Batalha, C. 1990, ApJ, 363, 654

Bessell, M. S., \& Brett, J. M. 1988, PASP, 100, 1134

Castelli, F., Gratton, R. G., \& Kurucz, R. L. 1997, A\&A, 318, 841

Chelli, A. 1999, A\&A, 349, L65

Cutri, R. M., Skrutskie, M. F., van Dyk, S., et al. 2003, 2MASS All Sky Catalog of point sources., eds. R. M. Cutri, M. F. Skrutskie, S. van Dyk, et al.

de Jager, C., \& Neven, L. 1959, The Observatory, 79, 102

Debosscher, J., Sarro, L. M., López, M., et al. 2009, A\&A, 506, 519

Deleuil, M., Meunier, J. C., Moutou, C., et al. 2009, AJ, 138, 649

Dias, W. S., Assafin, M., Flório, V., Alessi, B. S., \& Líbero, V. 2006, A\&A, 446, 949

Divan, L., \& Zorec, J. 1982, in Be Stars, eds. M. Jaschek, \& H.-G. Groth, IAU Symp., 98, 61

D’Odorico, S., Dekker, H., Mazzoleni, R., et al. 2006, in Proc. SPIE, 6269

Drimmel, R., Cabrera-Lavers, A., \& López-Corredoira, M. 2003, A\&A, 409, 205

Ekström, S., Meynet, G., Maeder, A., \& Barblan, F. 2008, A\&A, 478, 467

Ekström, S., Georgy, C., Eggenberger, P., et al. 2012, A\&A, 537, A146

Emilio, M., Andrade, L., Janot-Pacheco, E., et al. 2010, A\&A, 522, A43

Fabregat, J., \& Reglero, V. 1990, MNRAS, 247, 407

Fabregat, J., \& Torrejón, J. M. 2000, A\&A, 357, 451

Fabregat, J., Torrejon, J. M., Reig, P., et al. 1996, A\&AS, 119, 271

Floquet, M., Hubert, A. M., Hirata, R., et al. 2000, A\&A, 362, 1020

Frémat, Y., Zorec, J., Hubert, A. M., \& Floquet, M. 2005, A\&A, 440, 305

Frémat, Y., Neiner, C., Hubert, A. M., et al. 2006, A\&A, 451, 1053

Galazutdinov, G. A., Musaev, F. A., Krełowski, J., \& Walker, G. A. H. 2000,

PASP, 112, 648

Glass, I. S., Ellis, R., Huchra, J., et al. 1999, Handbook of Infrared Astronomy, eds. I. S. Glass, R. Ellis, J. Huchra, et al.

Gray, R. O., \& Corbally, C. J. 1994, AJ, 107, 742

Grevesse, N., \& Sauval, A. J. 1998, Space Sci. Rev., 85, 161

Gutiérrez-Soto, J. 2006, Ph.D. Thesis, Observatorio Astronomico de Valencia. Universidad de Valencia

Gutiérrez-Soto, J., Neiner, C., Fabregat, J., et al. 2011, in IAU Symp. 272, eds. C. Neiner, G. Wade, G. Meynet, \& G. Peters, 451

Hartigan, P., Hartmann, L., Kenyon, S., Hewett, R., \& Stauffer, J. 1989, ApJS, 70,899

Hawkins, F. M. 1961, MNRAS, 122, 285

Herbig, G. H. 1975, ApJ, 196, 129
Hernández, J., Calvet, N., Hartmann, L., et al. 2005, AJ, 129, 856 Huat, A.-L., Hubert, A. M., Baudin, F., et al. 2009, A\&A, 506, 95 Hubert, A. M., \& Floquet, M. 1998, A\&A, 335, 565

Hubeny, I., \& Lanz, T. 1995, ApJ, 439, 875

Hubert, A. M., Floquet, M., \& Zorec, J. 2000, in IAU Colloq. 175: The Be Phenomenon in Early-Type Stars, eds. M. A. Smith, H. F. Henrichs, \& J. Fabregat (San Francisco: ASP), 214, 48

Hubert-Delplace, A. M., Hubert, H., Ballereau, D., \& Chambon, M. T. 1982, in IAU Symp. 98, Be Stars, eds. M. Jaschek, \& H.-G. Groth, 195

Keller, S. C., Bessell, M. S., Cook, K. H., Geha, M., \& Syphers, D. 2002, AJ, 124,2039

Kohoutek, L., \& Wehmeyer, R. 1999, A\&AS, 134, 255

Kohoutek, L., \& Wehmeyer, R. 2003, Astron. Nachr., 324, 437

Kohoutek, L., \& Wehmeyer, R. 2004, Astron. Nachr., 325, 723

Kurucz, R. 1993, ATLAS9 Stellar Atmosphere Programs and $2 \mathrm{~km} \mathrm{~s}^{-1}$ grid. Kurucz CD-ROM No. 13 (Cambridge, Mass.: Smithsonian Astrophysical Observatory) 13

Lanz, T., \& Hubeny, I. 2003, in Stellar Atmosphere Modeling, eds. I. Hubeny, D. Mihalas, \& K. Werner (San Francisco: ASP), 288, 157

Maeder, A., Grebel, E. K., \& Mermilliod, J.-C. 1999, A\&A, 346, 459

Martayan, C., Frémat, Y., Hubert, A. M., et al. 2006, A\&A, 452, 273

Martayan, C., Frémat, Y., Hubert, A. M., et al. 2007, A\&A, 462, 683

Mathew, B., Subramaniam, A., \& Bhatt, B. C. 2008, MNRAS, 388, 1879

McSwain, M. V., \& Gies, D. R. 2005, ApJS, 161, 118

Meilland, A., Stee, P., Zorec, J., \& Kanaan, S. 2006, A\&A, 455, 953

Mennickent, R. E., Pietrzyński, G., Gieren, W., \& Szewczyk, O. 2002, A\&A, 393,887

Meyer, S. L. 1975, Data analysis for scientists and engineers, ed. S. L. Meyer

Meynet, G., \& Maeder, A. 2000, A\&A, 361, 101

Modigliani, A., Goldoni, P., Royer, F., et al. 2010, in Proc. SPIE, 7737

Moon, T. 1986, Ap\&SS, 122, 173

Moujtahid, A., Zorec, J., Hubert, A. M., Garcia, A., \& Burki, G. 1998, A\&AS, 129,289

Moujtahid, A., Zorec, J., \& Hubert, A. M. 1999, A\&A, 349, 151

Moujtahid, A., Zorec, J., \& Hubert, A. M. 2000a, in IAU Colloq. 175: The Be Phenomenon in Early-Type Stars, eds. M. A. Smith, H. F. Henrichs, \& J. Fabregat (San Francisco: ASP), 214, 506

Moujtahid, A., Zorec, J., \& Hubert, A. M. 2000b, in IAU Colloq. 175: The Be Phenomenon in Early-Type Stars, eds. M. A. Smith, H. F. Henrichs, \& J. Fabregat (San Francisco: ASP), 214, 510

Negueruela, I. 2004, Astron. Nachr., 325, 380

Neiner, C., Hubert, A. M., Frémat, Y., et al. 2003, A\&A, 409, 275

Okazaki, A. T. 1991, PASJ, 43, 75

Okazaki, A. T. 1997, A\&A, 318, 548

Pasquini, L., Avila, G., Blecha, A., et al. 2002, The Messenger, 110, 1

Pigulski, A., \& Kopacki, G. 2000, A\&AS, 146, 465

Pigulski, A., Kopacki, G., \& Kołaczkowski, Z. 2001, A\&A, 376, 144

Puga, E., Hony, S., Neiner, C., et al. 2009, A\&A, 503, 107

Robertson, T. H., \& Jordan, T. M. 1989, AJ, 98, 1354

Russeil, D. 2003, A\&A, 397, 133

Sale, S. E., Drew, J. E., Unruh, Y. C., et al. 2009, MNRAS, 392, 497

Sneden, C., Gehrz, R. D., Hackwell, J. A., York, D. G., \& Snow, T. P. 1978, ApJ, 223, 168

Snow, Jr., T. P., York, D. G., \& Welty, D. E. 1977, AJ, 82, 113

Stoeckley, T. R. 1968, MNRAS, 140, 141

Townsend, R. H. D., Owocki, S. P., \& Howarth, I. D. 2004, MNRAS, 350, 189

Vernet, J., Dekker, H., D'Odorico, S., et al. 2011, A\&A, 536, A105

Vinicius, M. M. F., Zorec, J., Leister, N. V., \& Levenhagen, R. S. 2006, A\&A, 446, 643

von Zeipel, H. 1924, MNRAS, 84, 665

Wisniewski, J. P., \& Bjorkman, K. S. 2006, ApJ, 652, 458

Witham, A. R., Knigge, C., Drew, J. E., et al. 2008, MNRAS, 384, 1277

Zorec, J. 1986, Structure et rotation differentielle dans les étoiles B avec et sans émission (Paris: Université Paris VII)

Zorec, J., \& Briot, D. 1997, A\&A, 318, 443

Zorec, J., \& Royer, F. 2012, A\&A, 537, A120

Zorec, J., Frémat, Y., \& Hubert, A. M. 2000, in IAU Colloq. 175: The Be Phenomenon in Early-Type Stars, eds. M. A. Smith, H. F. Henrichs, \& J. Fabregat (San Francisco: ASP), 214, 330

Zorec, J., Frémat, Y., \& Cidale, L. 2005, A\&A, 441, 235

Zorec, J., Frémat, Y., Domiciano de Souza, A., et al. 2011, A\&A, 526, A87

Pages 17 to 20 are available in the electronic edition of the journal at http://www . aanda. org 
T. Semaan et al.: Study of a sample of faint Be stars in the exofield of CoRoT. I.

\section{Appendix A: Tables of fundamental parameters}

Table A.1. Fundamental parameters and rotational frequencies corrected for rotational effects assuming that $\omega=\Omega / \Omega_{\mathrm{c}}=0.80$.

\begin{tabular}{cccccccccc}
\hline \hline \multirow{2}{*}{ CoRoT ID } & $N^{*}$ & $\begin{array}{c}\left\langle T_{\text {eff }}\right\rangle \\
\mathrm{K}\end{array}$ & $\begin{array}{c}\log \langle g\rangle \\
\mathrm{dex}\end{array}$ & $\begin{array}{c}v \sin i \\
\mathrm{~km} \mathrm{~s}^{-1}\end{array}$ & $\begin{array}{c}\log \langle L\rangle \pm \epsilon_{\log \langle L\rangle} \\
L_{\odot}\end{array}$ & $\begin{array}{c}R \pm \epsilon_{\mathrm{R}} \\
R_{\odot}\end{array}$ & $\begin{array}{c}M \pm \epsilon_{\mathrm{M}} \\
M_{\odot}\end{array}$ & $\begin{array}{c}\tau / \tau_{\mathrm{MS}} \pm \epsilon_{\tau / \tau} \mathrm{MS} \\
-\end{array}$ & $\begin{array}{c}v_{\mathrm{r}} \pm \epsilon_{v} \\
\mathrm{c} / \mathrm{d}\end{array}$ \\
\hline 101486436 & 1 & 12900 & 4.1 & 225 & $2.31 \pm 0.26$ & $2.9 \pm 0.6$ & $3.6 \pm 0.2$ & $0.51 \pm 0.19$ & $1.76 \pm 0.25$ \\
102595654 & 2 & 10800 & 3.5 & 325 & $2.85 \pm 0.27$ & $7.7 \pm 1.6$ & $4.2 \pm 0.3$ & $1.02 \pm 0.01$ & $1.06 \pm 0.26$ \\
102656190 & 4 & 20400 & 4.1 & 265 & $3.40 \pm 0.26$ & $4.1 \pm 0.8$ & $7.2 \pm 0.4$ & $0.37 \pm 0.25$ & $1.51 \pm 0.20$ \\
102672979 & 5 & 16100 & 3.5 & 209 & $3.69 \pm 0.28$ & $9.3 \pm 2.0$ & $7.1 \pm 0.5$ & $1.01 \pm 0.01$ & $0.46 \pm 0.07$ \\
102686433 & 6 & 17100 & 3.7 & 281 & $3.74 \pm 0.27$ & $8.7 \pm 1.8$ & $7.4 \pm 0.5$ & $0.99 \pm 0.03$ & $0.62 \pm 0.10$ \\
102719279 & 9 & 22300 & 3.7 & 277 & $4.25 \pm 0.28$ & $9.1 \pm 2.0$ & $11.1 \pm 0.9$ & $0.92 \pm 0.06$ & $0.60 \pm 0.09$ \\
102725623 & 11 & 13100 & 2.8 & 295 & $4.12 \pm 0.30$ & $22.9 \pm 5.3$ & $9.2 \pm 1.1$ & $1.01 \pm 0.01$ & $0.31 \pm 0.09$ \\
102728404 & 12 & 23400 & 4.0 & 194 & $3.86 \pm 0.27$ & $5.3 \pm 1.1$ & $9.6 \pm 0.6$ & $0.58 \pm 0.18$ & $1.11 \pm 0.15$ \\
102766835 & 14 & 33400 & 3.8 & 256 & $5.11 \pm 0.33$ & $11.0 \pm 2.8$ & $24.7 \pm 3.3$ & $0.68 \pm 0.09$ & $0.65 \pm 0.11$ \\
102785480 & 17 & 21700 & 3.9 & 319 & $4.05 \pm 0.27$ & $7.7 \pm 1.6$ & $9.9 \pm 0.7$ & $0.88 \pm 0.08$ & $0.79 \pm 0.12$ \\
102825808 & 18 & 20100 & 4.1 & 349 & $3.62 \pm 0.26$ & $5.5 \pm 1.1$ & $7.8 \pm 0.5$ & $0.74 \pm 0.13$ & $1.26 \pm 0.21$ \\
102829773 & 19 & 23400 & 4.3 & 396 & $3.50 \pm 0.25$ & $3.5 \pm 0.7$ & $8.4 \pm 0.5$ & $0.07 \pm 0.10$ & $2.27 \pm 0.33$ \\
102847615 & 20 & 17300 & 4.2 & 207 & $2.88 \pm 0.26$ & $3.1 \pm 0.6$ & $5.3 \pm 0.3$ & $0.23 \pm 0.21$ & $1.85 \pm 0.24$ \\
102904910 & 22 & 18600 & 4.1 & 278 & $3.30 \pm 0.26$ & $4.4 \pm 0.9$ & $6.5 \pm 0.4$ & $0.62 \pm 0.17$ & $1.36 \pm 0.19$ \\
\hline
\end{tabular}

Table A.2. Fundamental parameters and rotational frequencies corrected for rotational effects assuming that $\omega=\Omega / \Omega_{\mathrm{c}}=0.90$.

\begin{tabular}{|c|c|c|c|c|c|c|c|c|c|}
\hline CoRoT ID & $N^{*}$ & $\begin{array}{c}\left\langle T_{\text {eff }}\right\rangle \\
\mathrm{K}\end{array}$ & $\begin{array}{c}\log \langle g\rangle \\
\operatorname{dex}\end{array}$ & $\begin{array}{c}v \sin i \\
\mathrm{~km} \mathrm{~s}^{-1}\end{array}$ & $\begin{array}{c}\log \langle L\rangle \pm \epsilon_{\log \langle L\rangle} \\
L_{\odot}\end{array}$ & $\begin{array}{l}R \pm \epsilon_{\mathrm{R}} \\
R_{\odot}\end{array}$ & $\begin{array}{c}M \pm \epsilon_{\mathrm{M}} \\
M_{\odot}\end{array}$ & $\begin{array}{c}\tau / \tau_{\mathrm{MS}} \pm \epsilon_{\tau / \tau_{\mathrm{MS}}} \\
-\end{array}$ & $\begin{array}{c}v_{\mathrm{r}} \pm \epsilon_{v} \\
\mathrm{c} / \mathrm{d}\end{array}$ \\
\hline 101486436 & 1 & 12900 & 4.1 & 224 & $2.26 \pm 0.26$ & $2.7 \pm 0.6$ & $3.5 \pm 0.2$ & $0.36 \pm 0.24$ & $2.00 \pm 0.25$ \\
\hline 102595654 & 2 & 10300 & 3.4 & 327 & $2.72 \pm 0.27$ & $7.4 \pm 1.6$ & $3.9 \pm 0.3$ & $1.02 \pm 0.005$ & $1.30 \pm 0.30$ \\
\hline 102656190 & 4 & 20500 & 4.1 & 272 & $3.35 \pm 0.26$ & $3.9 \pm 0.8$ & $7.1 \pm 0.4$ & $0.29 \pm 0.23$ & $1.72 \pm 0.21$ \\
\hline 102672979 & 5 & 16100 & 3.5 & 216 & $3.65 \pm 0.28$ & $8.7 \pm 1.9$ & $6.9 \pm 0.5$ & $1.00 \pm 0.02$ & $0.53 \pm 0.07$ \\
\hline 102686433 & 6 & 17300 & 3.7 & 284 & $3.69 \pm 0.27$ & $8.0 \pm 1.7$ & $7.3 \pm 0.5$ & $0.98 \pm 0.04$ & $0.70 \pm 0.10$ \\
\hline 102719279 & 9 & 22300 & 3.7 & 282 & $4.19 \pm 0.28$ & $8.5 \pm 1.8$ & $10.8 \pm 0.8$ & $0.90 \pm 0.07$ & $0.69 \pm 0.09$ \\
\hline 102725623 & 11 & 12900 & 2.8 & 298 & $4.05 \pm 0.30$ & $21.9 \pm 5.1$ & $8.8 \pm 1.0$ & $1.02 \pm 0.005$ & $0.35 \pm 0.09$ \\
\hline 102728404 & 12 & 23500 & 4.0 & 197 & $3.81 \pm 0.27$ & $5.0 \pm 1.0$ & $9.4 \pm 0.5$ & $0.52 \pm 0$ & $1.30 \pm 0.16$ \\
\hline & 14 & 400 & 3.8 & 259 & $5.05 \pm$ & $10.4 \pm$ & 23.7 & $0.65=$ & $0.76 \pm 0.12$ \\
\hline 1027 & 17 & 21800 & 3.8 & 326 & $4.01 \pm 0.27$ & $7.2 \pm 1.5$ & $9.7 \pm 0.7$ & $0.84 \pm 0.09$ & $0.90 \pm 0.12$ \\
\hline 102825808 & 18 & 20300 & 4.1 & 357 & & $5.1 \pm 1.1$ & $7.7 \pm 0.5$ & $0.68 \pm 0.15$ & $1.41 \pm 0.20$ \\
\hline 102829773 & 19 & 500 & 4.3 & 403 & $3.46 \pm 0.25$ & $3.3 \pm 0.7$ & $8.3 \pm 0.4$ & $0.04 \pm 0.06$ & $2.55 \pm 0.32$ \\
\hline 102 & 20 & 7400 & 4.2 & 213 & $2.83 \pm 0.26$ & $2.9 \pm 0.6$ & $5.2 \pm 0.3$ & $0.16 \pm 0.17$ & $2.14 \pm 0.25$ \\
\hline 102904910 & 22 & 18700 & 4.1 & 287 & $3.26 \pm 0.26$ & $4.1 \pm 0.8$ & $6.4 \pm 0.3$ & $0.55 \pm 0.19$ & $1.53 \pm 0.19$ \\
\hline
\end{tabular}

Table A.3. Fundamental parameters and rotational frequencies corrected for rotational effects assuming that $\omega=\Omega / \Omega_{\mathrm{c}}=0.98$.

\begin{tabular}{cccccccccc}
\hline \hline CoRoT ID & $N^{*}$ & $\begin{array}{c}\left\langle T_{\text {eff }}\right\rangle \\
\mathrm{K}\end{array}$ & $\begin{array}{c}\log \langle g\rangle \\
\mathrm{dex}\end{array}$ & $\begin{array}{c}v \sin i \\
\mathrm{~km} \mathrm{~s}^{-1}\end{array}$ & $\begin{array}{c}\log \langle L\rangle \pm \epsilon_{\log \langle L\rangle} \\
L_{\odot}\end{array}$ & $\begin{array}{c}R \pm \epsilon_{R} \\
R_{\odot}\end{array}$ & $\begin{array}{c}M \pm \epsilon_{\mathrm{M}} \\
M_{\odot}\end{array}$ & $\begin{array}{c}\tau / \tau_{\mathrm{MS}} \pm \epsilon_{\tau / \tau} \mathrm{MS} \\
-\end{array}$ & $\begin{array}{c}v_{\mathrm{r}} \pm \epsilon_{v} \\
\mathrm{c} / \mathrm{d}\end{array}$ \\
\hline 101486436 & 1 & 13200 & 4.1 & 260 & $2.23 \pm 0.26$ & $2.5 \pm 0.5$ & $3.5 \pm 0.2$ & $0.25 \pm 0.21$ & $2.19 \pm 0.23$ \\
102595654 & 2 & 11400 & 3.5 & 327 & $2.85 \pm 0.27$ & $7.0 \pm 1.5$ & $4.2 \pm 0.3$ & $1.01 \pm 0.01$ & $1.17 \pm 0.20$ \\
102656190 & 4 & 20600 & 4.1 & 288 & $3.29 \pm 0.26$ & $3.5 \pm 0.7$ & $7.0 \pm 0.4$ & $0.17 \pm 0.18$ & $1.88 \pm 0.19$ \\
102672979 & 5 & 16300 & 3.4 & 233 & $3.59 \pm 0.28$ & $8.0 \pm 1.7$ & $6.8 \pm 0.5$ & $0.99 \pm 0.04$ & $0.57 \pm 0.07$ \\
102686433 & 6 & 17900 & 3.6 & 321 & $3.69 \pm 0.27$ & $7.5 \pm 1.6$ & $7.4 \pm 0.5$ & $0.95 \pm 0.06$ & $0.74 \pm 0.09$ \\
102719279 & 9 & 22300 & 3.7 & 295 & $4.12 \pm 0.28$ & $7.9 \pm 1.7$ & $10.4 \pm 0.8$ & $0.87 \pm 0.08$ & $0.76 \pm 0.08$ \\
102725623 & 11 & 13900 & 2.8 & 298 & $4.15 \pm 0.30$ & $21.2 \pm 4.9$ & $9.5 \pm 1.0$ & $1.01 \pm 0.01$ & $0.37 \pm 0.08$ \\
102728404 & 12 & 23700 & 4.0 & 207 & $3.75 \pm 0.27$ & $4.5 \pm 0.9$ & $9.3 \pm 0.5$ & $0.32 \pm 0.24$ & $1.46 \pm 0.15$ \\
102766835 & 14 & 33600 & 3.8 & 267 & $4.98 \pm 0.33$ & $9.4 \pm 2.4$ & $22.7 \pm 2.6$ & $0.60 \pm 0.13$ & $0.87 \pm 0.12$ \\
102785480 & 17 & 21600 & 3.8 & 338 & $3.91 \pm 0.27$ & $6.6 \pm 1.4$ & $9.2 \pm 0.6$ & $0.81 \pm 0.10$ & $1.00 \pm 0.12$ \\
102825808 & 18 & 20300 & 4.0 & 383 & $3.52 \pm 0.26$ & $4.7 \pm 1.0$ & $7.5 \pm 0.4$ & $0.61 \pm 0.17$ & $1.54 \pm 0.19$ \\
102829773 & 19 & 23500 & 4.3 & 418 & $3.39 \pm 0.25$ & $3.0 \pm 0.6$ & $8.1 \pm 0.4$ & $0.01 \pm 0.03$ & $2.71 \pm 0.28$ \\
102847615 & 20 & 17600 & 4.2 & 231 & $2.78 \pm 0.26$ & $2.7 \pm 0.5$ & $5.1 \pm 0.3$ & $0.08 \pm 0.11$ & $2.38 \pm 0.23$ \\
102904910 & 22 & 18900 & 4.0 & 310 & $3.20 \pm 0.26$ & $3.8 \pm 0.8$ & $6.3 \pm 0.3$ & $0.37 \pm 0.25$ & $1.64 \pm 0.17$ \\
\hline
\end{tabular}


A\&A 551, A130 (2013)

Table A.4. Fundamental parameters and rotational frequencies corrected for rotational effects assuming that $\omega=\Omega / \Omega_{\mathrm{c}}=0.999$.

\begin{tabular}{|c|c|c|c|c|c|c|c|c|c|}
\hline CoRoT ID & $\mathrm{N}^{*}$ & $\begin{array}{c}\left\langle T_{\text {eff }}\right\rangle \\
\mathrm{K}\end{array}$ & $\begin{array}{c}\log \langle g\rangle \\
\operatorname{dex}\end{array}$ & $\begin{array}{c}v \sin i \\
\mathrm{~km} \mathrm{~s}^{-1}\end{array}$ & $\begin{array}{c}\log \langle L\rangle \pm \epsilon_{\log \langle L\rangle} \\
L_{\odot}\end{array}$ & $\begin{array}{c}R \pm \epsilon_{\mathrm{R}} \\
R_{\odot}\end{array}$ & $\begin{array}{c}M \pm \epsilon_{\mathrm{M}} \\
M_{\odot}\end{array}$ & $\begin{array}{c}\tau / \tau_{\mathrm{MS}} \pm \epsilon_{\tau / \tau_{\mathrm{MS}}} \\
-\end{array}$ & $\begin{array}{c}\nu_{\mathrm{r}} \pm \epsilon_{v} \\
\mathrm{c} / \mathrm{d}\end{array}$ \\
\hline 101486436 & 1 & 13300 & 4.1 & 271 & $2.22 \pm 0.26$ & $2.5 \pm 0.5$ & $3.5 \pm 0.2$ & $0.22 \pm 0.20$ & $2.20 \pm 0.20$ \\
\hline 102595654 & 2 & 11900 & 3.6 & 327 & $2.91 \pm 0.27$ & $6.9 \pm 1.5$ & $4.4 \pm 0.3$ & $1.01 \pm 0.02$ & $0.99 \pm 0.13$ \\
\hline 102656190 & 4 & 20700 & 4.1 & 295 & $3.27 \pm 0.26$ & $3.4 \pm 0.7$ & $7.0 \pm 0.4$ & $0.14 \pm 0.16$ & $1.90 \pm 0.17$ \\
\hline 102672979 & 5 & 16400 & 3.4 & 240 & $3.58 \pm 0.28$ & $7.8 \pm 1.7$ & $6.7 \pm 0.5$ & $0.98 \pm 0.04$ & $0.57 \pm 0.06$ \\
\hline 102686433 & 6 & 18000 & 3.6 & 334 & $3.69 \pm 0.27$ & $7.3 \pm 1.6$ & $7.5 \pm 0.5$ & $0.94 \pm 0.06$ & $0.73 \pm 0.07$ \\
\hline 102719279 & 9 & 22200 & 3.6 & 299 & $4.10 \pm 0.28$ & $7.7 \pm 1.7$ & $10.3 \pm 0.8$ & $0.86 \pm 0.08$ & $0.77 \pm 0.08$ \\
\hline 102725623 & 11 & 14400 & 2.9 & 299 & $4.20 \pm 0.30$ & $20.9 \pm 4.9$ & $9.8 \pm 1.0$ & $1.01 \pm 0.01$ & $0.37 \pm 0.07$ \\
\hline 102728404 & 12 & 23800 & 4.0 & 211 & $3.73 \pm 0.27$ & $4.4 \pm 0.9$ & $9.3 \pm 0.5$ & $0.28 \pm 0.23$ & $1.48 \pm 0.13$ \\
\hline 102766835 & 14 & 33700 & 3.8 & 270 & $4.96 \pm 0.33$ & $9.2 \pm 2.3$ & $22.5 \pm 2.5$ & $0.58 \pm 0.14$ & $0.89 \pm 0.10$ \\
\hline 102785480 & 17 & 21500 & 3.8 & 343 & $3.88 \pm 0.27$ & $6.5 \pm 1.4$ & $9.1 \pm 0.6$ & $0.80 \pm 0.10$ & $1.02 \pm 0.10$ \\
\hline 102825808 & 18 & 20200 & 4.0 & 392 & $3.49 \pm 0.26$ & $4.7 \pm 1.0$ & $7.4 \pm 0.4$ & $0.60 \pm 0.18$ & $1.57 \pm 0.17$ \\
\hline 102829773 & 19 & 23500 & 4.3 & 424 & $3.37 \pm 0.25$ & $3.0 \pm 0.6$ & $8.0 \pm 0.4$ & $0.01 \pm 0.03$ & $2.72 \pm 0.25$ \\
\hline 102847615 & 20 & 17700 & 4.2 & 239 & $2.76 \pm 0.26$ & $2.6 \pm 0.5$ & $5.1 \pm 0.3$ & $0.06 \pm 0.09$ & $2.42 \pm 0.21$ \\
\hline 102904910 & 22 & 19000 & 4.0 & 318 & $3.19 \pm 0.26$ & $3.7 \pm 0.8$ & $6.3 \pm 0.3$ & $0.34 \pm 0.25$ & $1.64 \pm 0.15$ \\
\hline
\end{tabular}

Table A.5. Fundamental parameters and rotational frequencies corrected for rotational effects assuming that $\omega=\Omega / \Omega_{\mathrm{c}}=0.9999$.

\begin{tabular}{|c|c|c|c|c|c|c|c|c|c|}
\hline CoRoT ID & $N^{*}$ & $\begin{array}{c}\left\langle T_{\text {eff }}\right\rangle \\
\mathrm{K} \\
\end{array}$ & $\begin{array}{c}\log \langle g\rangle \\
\operatorname{dex}\end{array}$ & $\begin{array}{c}v \sin i \\
\mathrm{~km} \mathrm{~s}^{-1}\end{array}$ & $\begin{array}{c}\log \langle L\rangle \pm \epsilon_{\log \langle L\rangle} \\
L_{\odot}\end{array}$ & $\begin{array}{c}R \pm \epsilon_{\mathrm{R}} \\
R_{\odot}\end{array}$ & $\begin{array}{c}M \pm \epsilon_{\mathrm{M}} \\
M_{\odot}\end{array}$ & $\begin{array}{c}\tau / \tau_{\mathrm{MS}} \pm \epsilon_{\tau / \tau_{\mathrm{MS}}} \\
-\end{array}$ & $\begin{array}{c}v_{\mathrm{r}} \pm \epsilon_{v} \\
\mathrm{c} / \mathrm{d}\end{array}$ \\
\hline 101486436 & 1 & 13300 & 4.1 & 272 & $2.22 \pm 0.26$ & $2.5 \pm 0.5$ & $3.5 \pm 0.2$ & $0.22 \pm 0.20$ & $2.20 \pm 0.19$ \\
\hline 102595654 & 2 & 11900 & 3.6 & 327 & $2.92 \pm 0.27$ & $6.9 \pm 1.5$ & $4.4 \pm 0.3$ & $1.01 \pm 0.02$ & $0.98 \pm 0.13$ \\
\hline 102656190 & 4 & 20700 & 4.1 & 295 & $3.27 \pm 0.26$ & $3.4 \pm 0.7$ & $7.0 \pm 0.4$ & $0.14 \pm 0.16$ & $1.89 \pm 0.16$ \\
\hline 102672979 & 5 & 16400 & 3.4 & 240 & $3.58 \pm 0.28$ & $7.8 \pm 1.7$ & $6.7 \pm 0.5$ & $0.98 \pm 0.04$ & $0.56 \pm 0.05$ \\
\hline 102686433 & 6 & 18100 & 3.6 & 335 & $3.69 \pm 0.27$ & $7.3 \pm 1.6$ & $7.5 \pm 0.5$ & $0.94 \pm 0.06$ & $0.72 \pm 0.07$ \\
\hline 102719279 & 9 & 22200 & 3.6 & 300 & $4.10 \pm 0.28$ & $7.7 \pm 1.7$ & $10.3 \pm 0.8$ & $0.86 \pm 0.08$ & $0.77 \pm 0.07$ \\
\hline 102725623 & 11 & 14400 & 2.9 & 299 & $4.21 \pm 0.30$ & $20.9 \pm 4.9$ & $9.8 \pm 1.0$ & $1.01 \pm 0.01$ & $0.36 \pm 0.07$ \\
\hline 102728404 & 12 & 23900 & 4.0 & 211 & $3.73 \pm 0.27$ & $4.4 \pm 0.9$ & $9.3 \pm 0.5$ & $0.28 \pm 0.23$ & $1.48 \pm 0.13$ \\
\hline 102766835 & 14 & 33700 & 3.8 & 270 & $4.96 \pm 0.33$ & $9.2 \pm 2.3$ & $22.5 \pm 2.5$ & $0.58 \pm 0.14$ & $0.88 \pm 0.10$ \\
\hline 102785480 & 17 & 21500 & 3.7 & 343 & $3.88 \pm 0.27$ & $6.5 \pm 1.4$ & $9.1 \pm 0.6$ & $0.80 \pm 0.10$ & $1.01 \pm 0.10$ \\
\hline 102825808 & 18 & 20200 & 4.0 & 393 & $3.49 \pm 0.26$ & $4.6 \pm 1.0$ & $7.4 \pm 0.4$ & $0.60 \pm 0.18$ & $1.57 \pm 0.16$ \\
\hline 102829773 & 19 & 23500 & 4.3 & 425 & $3.37 \pm 0.25$ & $3.0 \pm 0.6$ & $8.0 \pm 0.4$ & $0.01 \pm 0.03$ & $2.70 \pm 0.24$ \\
\hline 102847615 & 20 & 17700 & 4.2 & 239 & $2.76 \pm 0.26$ & $2.6 \pm 0.5$ & $5.1 \pm 0.3$ & $0.06 \pm 0.09$ & $2.40 \pm 0.20$ \\
\hline 102904910 & 22 & 19000 & 4.0 & 318 & $3.19 \pm 0.26$ & $3.7 \pm 0.8$ & $6.3 \pm 0.3$ & $0.33 \pm 0.24$ & $1.63 \pm 0.14$ \\
\hline
\end{tabular}

Table A.6. Fundamental parameters and rotational frequencies corrected for rotational effects assuming that $\omega=\Omega / \Omega_{\mathrm{c}}=1.0$.

\begin{tabular}{cccccccccc}
\hline \hline CoRoT ID & $N^{*}$ & $\begin{array}{c}\left\langle T_{\text {eff }}\right\rangle \\
\mathrm{K}\end{array}$ & $\begin{array}{c}\log \langle g\rangle \\
\mathrm{dex}\end{array}$ & $\begin{array}{c}v \sin i \\
\mathrm{~km} \mathrm{~s}^{-1}\end{array}$ & $\begin{array}{c}\log \langle L\rangle \pm \epsilon_{\log \langle L\rangle} \\
L_{\odot}\end{array}$ & $\begin{array}{c}R \pm \epsilon_{\mathrm{R}} \\
R_{\odot}\end{array}$ & $\begin{array}{c}M \pm \epsilon_{\mathrm{M}} \\
M_{\odot}\end{array}$ & $\begin{array}{c}\tau / \tau_{\mathrm{MS}} \pm \epsilon_{\tau / \tau} \mathrm{MS} \\
-\end{array}$ & $\begin{array}{c}v_{\mathrm{r}} \pm \epsilon_{v} \\
\mathrm{c} / \mathrm{d}\end{array}$ \\
\hline 101486436 & 1 & 13300 & 4.1 & 272 & $2.22 \pm 0.26$ & $2.5 \pm 0.5$ & $3.5 \pm 0.2$ & $0.22 \pm 0.20$ & $2.19 \pm 0.19$ \\
102595654 & 2 & 11900 & 3.6 & 327 & $2.92 \pm 0.27$ & $6.9 \pm 1.5$ & $4.4 \pm 0.3$ & $1.01 \pm 0.02$ & $0.97 \pm 0.13$ \\
102656190 & 4 & 20700 & 4.1 & 295 & $3.27 \pm 0.26$ & $3.4 \pm 0.7$ & $7.0 \pm 0.4$ & $0.14 \pm 0.16$ & $1.89 \pm 0.16$ \\
102672979 & 5 & 16400 & 3.4 & 240 & $3.58 \pm 0.28$ & $7.8 \pm 1.7$ & $6.7 \pm 0.5$ & $0.98 \pm 0.04$ & $0.56 \pm 0.05$ \\
102686433 & 6 & 18100 & 3.6 & 335 & $3.69 \pm 0.27$ & $7.3 \pm 1.6$ & $7.5 \pm 0.5$ & $0.94 \pm 0.06$ & $0.72 \pm 0.07$ \\
102719279 & 9 & 22200 & 3.6 & 300 & $4.10 \pm 0.28$ & $7.7 \pm 1.7$ & $10.3 \pm 0.8$ & $0.86 \pm 0.08$ & $0.77 \pm 0.07$ \\
102725623 & 11 & 14400 & 2.9 & 299 & $4.21 \pm 0.30$ & $20.9 \pm 4.9$ & $9.9 \pm 1.0$ & $1.01 \pm 0.01$ & $0.36 \pm 0.06$ \\
102728404 & 12 & 23900 & 4.0 & 212 & $3.73 \pm 0.27$ & $4.4 \pm 0.9$ & $9.3 \pm 0.5$ & $0.28 \pm 0.23$ & $1.47 \pm 0.12$ \\
102766835 & 14 & 33700 & 3.8 & 270 & $4.96 \pm 0.33$ & $9.1 \pm 2.3$ & $22.5 \pm 2.5$ & $0.58 \pm 0.14$ & $0.88 \pm 0.10$ \\
102785480 & 17 & 21500 & 3.7 & 343 & $3.88 \pm 0.27$ & $6.5 \pm 1.4$ & $9.1 \pm 0.6$ & $0.80 \pm 0.10$ & $1.01 \pm 0.10$ \\
102825808 & 18 & 20200 & 4.0 & 393 & $3.49 \pm 0.26$ & $4.6 \pm 1.0$ & $7.4 \pm 0.4$ & $0.60 \pm 0.18$ & $1.56 \pm 0.16$ \\
102829773 & 19 & 23500 & 4.3 & 425 & $3.37 \pm 0.25$ & $3.0 \pm 0.6$ & $8.0 \pm 0.4$ & $0.01 \pm 0.03$ & $2.69 \pm 0.23$ \\
102847615 & 20 & 17700 & 4.2 & 239 & $2.76 \pm 0.26$ & $2.6 \pm 0.5$ & $5.1 \pm 0.3$ & $0.06 \pm 0.08$ & $2.40 \pm 0.20$ \\
102904910 & 22 & 19000 & 4.0 & 318 & $3.19 \pm 0.26$ & $3.7 \pm 0.8$ & $6.3 \pm 0.3$ & $0.33 \pm 0.24$ & $1.63 \pm 0.14$ \\
\hline
\end{tabular}


T. Semaan et al.: Study of a sample of faint Be stars in the exofield of CoRoT. I.

\section{Appendix B: Figures of different fits}
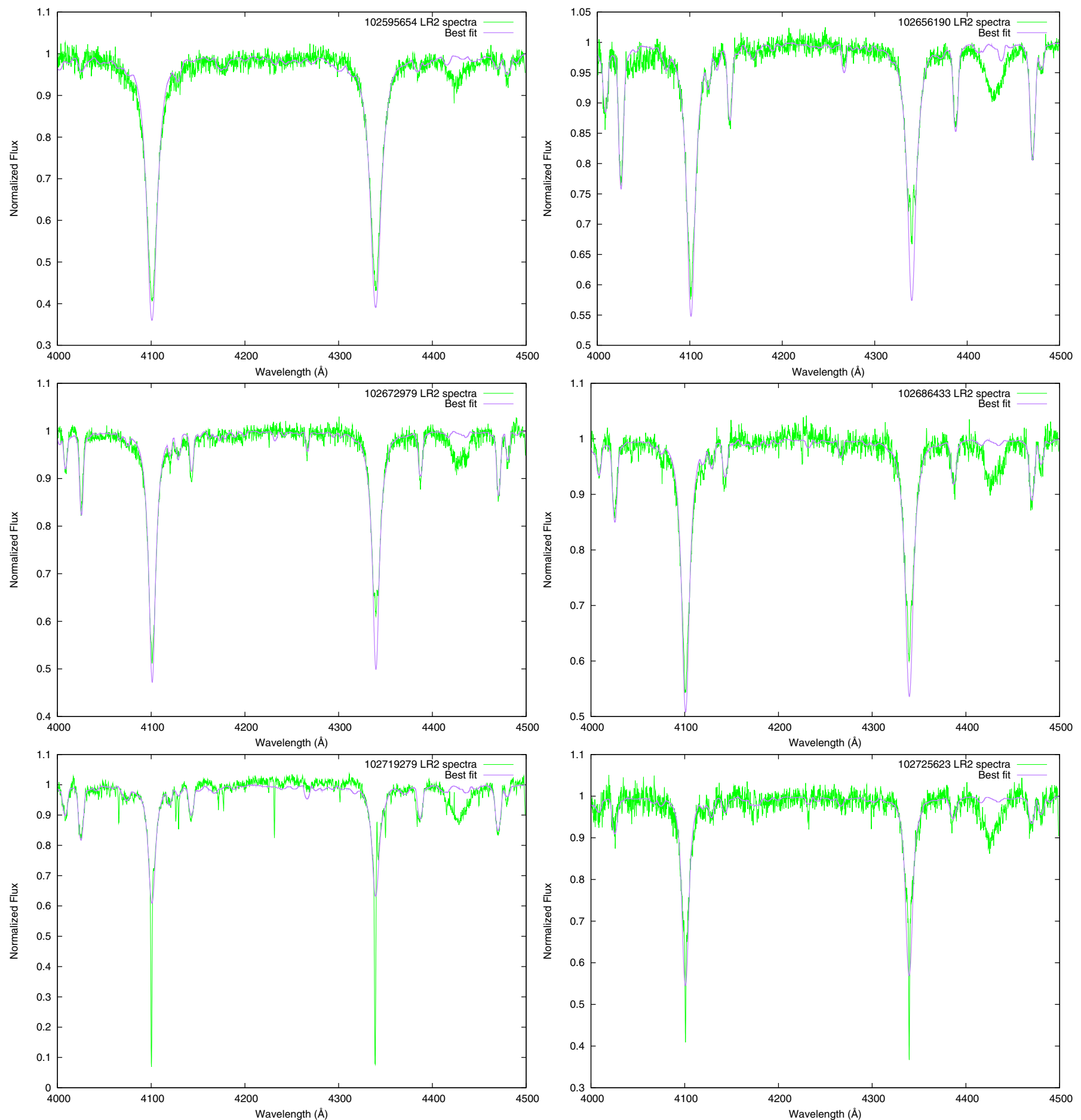

Fig. B.1. Fit of the observed spectra corrected for the veiling effect if needed $(r>1.25)$ of the stars CoRoT ID 102595654, 102656190, 102672979, $102686433,102719279,102725623$ with GIRFIT in the wavelength interval $\lambda \lambda 4000-4500 \AA$. 
A\&A 551, A130 (2013)
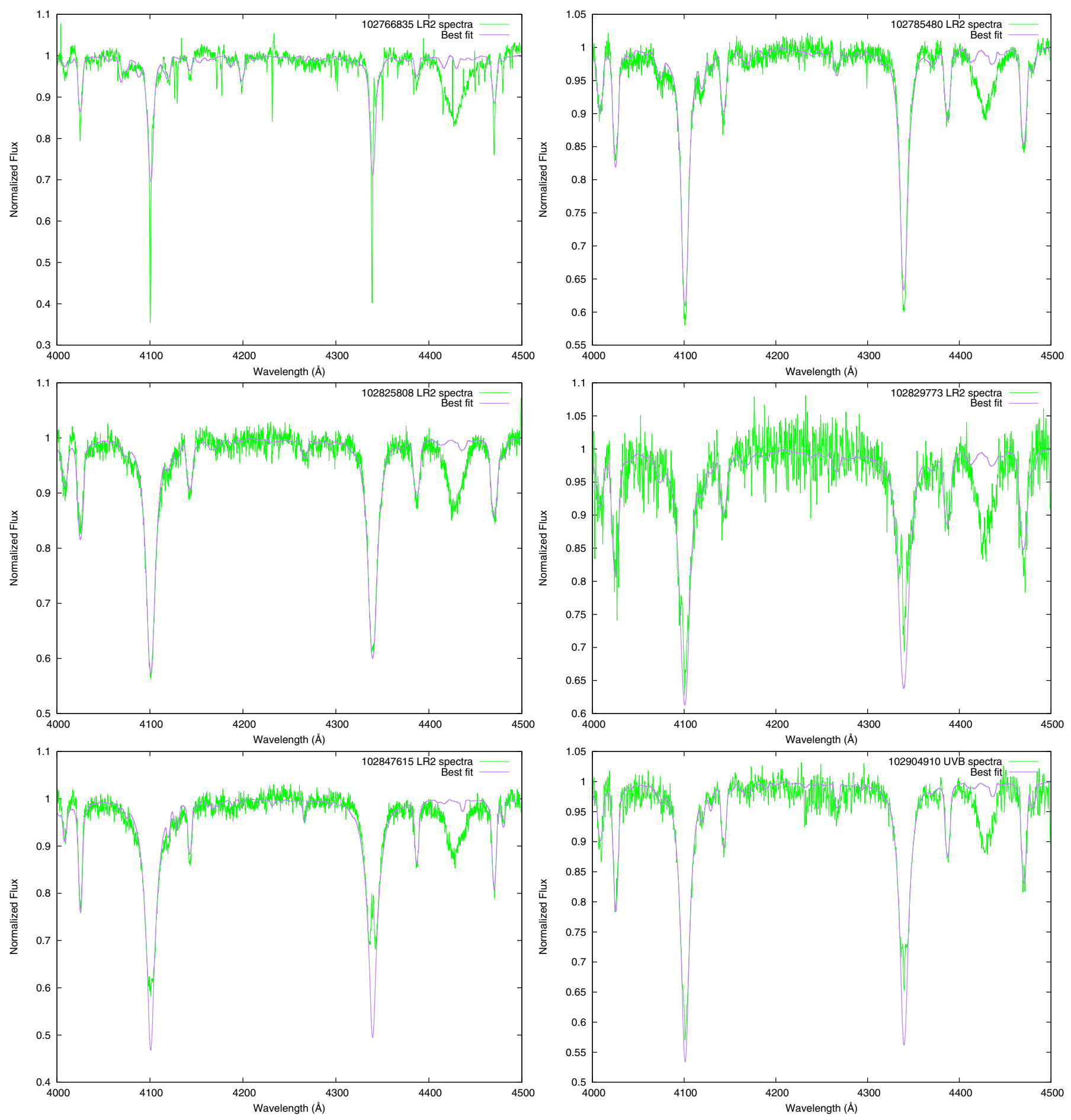

Fig. B.2. Fit of the observed spectra corrected for the veiling effect if needed $(r>1.25)$ of the stars CoRoT ID 102766835, 102785480, 102825808, $102829773,102847615,102904910$ with GIRFIT in the wavelength interval $\lambda \lambda 4000-4500 \AA$. 